\title{
Sox17 promotes tumor angiogenesis and destabilizes tumor vessels in mice
}

\author{
Hanseul Yang, ${ }^{1}$ Sungsu Lee, ${ }^{1}$ Seungjoo Lee, ${ }^{1}$ Kangsan Kim, ${ }^{1}$ Yeseul Yang, ${ }^{1}$ Jeong Hoon Kim, ${ }^{2}$ \\ Ralf H. Adams, ${ }^{3,4}$ James M. Wells, ${ }^{5}$ Sean J. Morrison, ${ }^{6}$ Gou Young Koh, ${ }^{1}$ and Injune Kim ${ }^{1}$ \\ ${ }^{1}$ Graduate School of Medical Science and Engineering, Korea Advanced Institute of Science and Technology, Daejeon, Republic of Korea. \\ 2Department of Neurological Surgery, Asan Medical Center, College of Medicine, University of Ulsan, Seoul, Republic of Korea. \\ ${ }^{3}$ Department of Tissue Morphogenesis, Max Plank Institute for Molecular Biomedicine, Münster, Germany. ${ }^{4}$ Faculty of Medicine, University of Münster, \\ Münster, Germany. ${ }^{D}$ Division of Developmental Biology, Cincinnati Children's Hospital Medical Center, Cincinnati, Ohio, USA. \\ ${ }^{6}$ Howard Hughes Medical Institute, University Texas Southwestern Medical Center, Dallas, Texas, USA.
}

\begin{abstract}
Little is known about the transcriptional regulation of tumor angiogenesis, and tumor ECs (tECs) remain poorly characterized. Here, we studied the expression pattern of the transcription factor Sox 17 in the vasculature of murine and human tumors and investigated the function of Sox 17 during tumor angiogenesis using Sox 17 genetic mouse models. Sox 17 was specifically expressed in tECs in a heterogeneous pattern; in particular, strong Sox17 expression distinguished tECs with high VEGFR2 expression. Whereas overexpression of Sox 17 in tECs promoted tumor angiogenesis and vascular abnormalities, Sox17 deletion in tECs reduced tumor angiogenesis and normalized tumor vessels, inhibiting tumor growth. Tumor vessel normalization by Sox17 deletion was long lasting, improved anticancer drug delivery into tumors, and inhibited tumor metastasis. Sox17 promoted endothelial sprouting behavior and upregulated VEGFR2 expression in a cell-intrinsic manner. Moreover, Sox 17 increased the percentage of tumor-associated CD11b $\mathrm{b}^{+} \mathrm{Gr}-\mathbf{1}^{+}$myeloid cells within tumors. The vascular effects of Sox 17 persisted throughout tumor growth. Interestingly, Sox17 expression specific to tECs was also observed in highly vascularized human glioblastoma samples. Our findings establish Sox17 as a key regulator of tumor angiogenesis and tumor progression.
\end{abstract}

\section{Introduction}

In the last few decades, regulation of tumor angiogenesis has been central to the fields of vascular biology and oncology, as tumor growth critically depends on new blood vessel formation. Tumor angiogenesis, characterized by excessive angiogenesis and abnormal morphogenesis, is mediated by multiple angiogenic regulators $(1,2)$. Many of the angiogenic regulators implicated in tumor angiogenesis are surface receptors on ECs and their cognate ligands being expressed by the tumor cells and tumor stromal cells. The key ligands and receptors, including VEGF/VEGFRs and the angiopoietin and Tie2 pathways, have been the main targets of therapies seeking to control tumor angiogenesis (3-5). Despite therapeutic success for some cancers, blockade of these pathways occasionally results in incomplete inhibition or evasive activation of tumor angiogenesis (6-9), emphasizing the need for a more complete understanding of angiogenic regulation.

Compared with information from the many studies on growth factors, their receptors, and the subsequent signaling pathways that govern tumor angiogenesis, little is known about transcriptional regulation of tumor angiogenesis. For example, the transcriptional regulation of VEGFR2, which encodes the most important receptor for angiogenesis, remains elusive in tumor angiogenesis, although many studies have investigated VEGFR2 expression in vascular development (10-12). Tumor angiogenesis is a continuous process $(2,4)$; thus, transcription factors that elicit a delayed response are expected to function as long-term regulators of tumor angiogenesis. However, the roles of transcription factors such as Etv2, COUF-TFII, and Prox-1 (13-15), which are crucial for vascular development, have

Authorship note: Hanseul Yang and Sungsu Lee contributed equally to this work. Conflict of interest: The authors have declared that no conflict of interest exists. Citation for this article: J Clin Invest. 2013;123(1):418-431. doi:10.1172/JCI64547. not been well studied in the context of tumor angiogenesis. More studies on the transcription factors are needed to clarify transcriptional regulation of key players in tumor angiogenesis.

Although tumor vessel morphogenesis is influenced by various cells within the tumor (4), tumor ECs (tECs) may represent one of the most important cell types. Nonetheless, it is not completely clear how the characteristics of tECs differ from those of normal ECs, and the properties of tECs are extrapolated from studies on ECs in vascular development. Although different ECs coexist in vascular development, as shown in tip, stalk, and phalanx cells that are distinct from each other in morphology and signaling characteristics (16-19), the heterogeneity of tECs has never been studied at the individual cell level. Analysis of tEC heterogeneity is important to better understand the mechanisms of tumor angiogenesis.

Sox17 is a transcription factor belonging to the Sox family, which contains HMG box (20). Although Sox17 has been considered an important regulator in biological processes, including endoderm formation during gastrulation (21), segregation of ventral foregut lineages (22), and maintenance of fetal identity in HSCs (23), its significance in vascular biology is only beginning to be recognized. In the context of the vascular system, Sox 17 expression is observed specifically in ECs during vascular development (24). Although global deletion of Sox 17 results in abnormal vascular development, the contribution of Sox17 to endothelial function remains unclear (25). In the human genome, the Sox17 locus is one of the most susceptible loci associated with intracranial aneurysms, suggesting its importance for blood vessel maintenance (26). However, the expression and function of Sox 17 in tumor angiogenesis have not been investigated.

In this study, we found profoundly increased Sox 17 expression in tECs during tumor progression. Using Sox17 genetic mouse models, we provide compelling evidence that endothelial Sox17 has both an intrinsic effect on tECs and an extrinsic effect on the peri- 

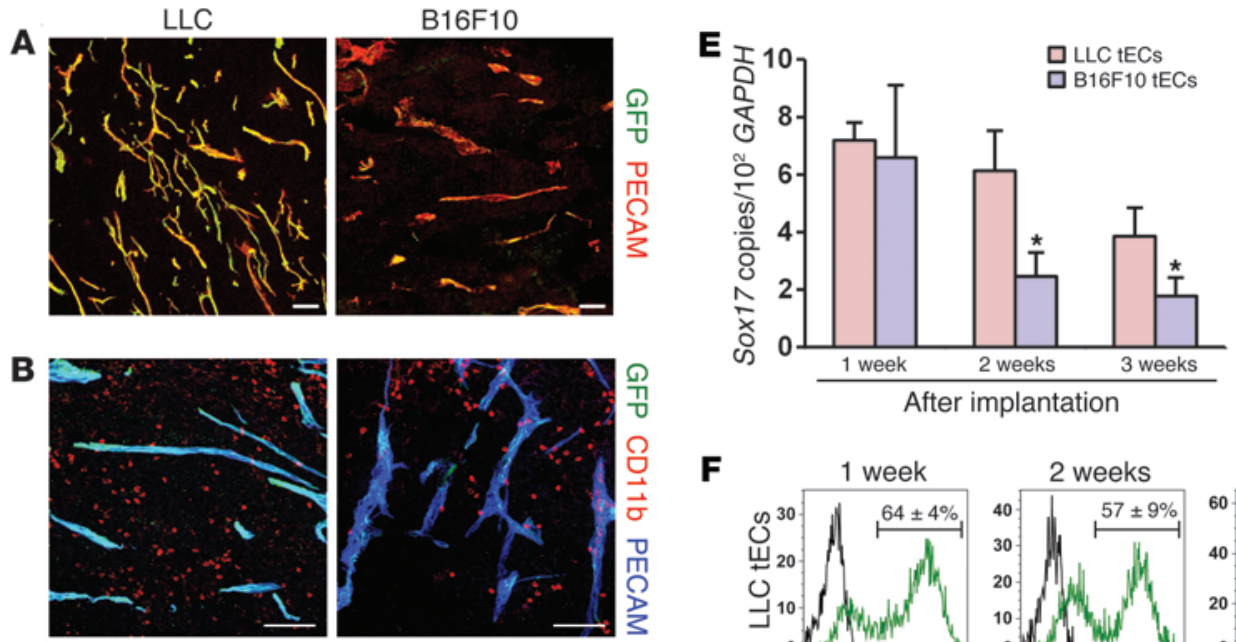

$\mathbf{F}$
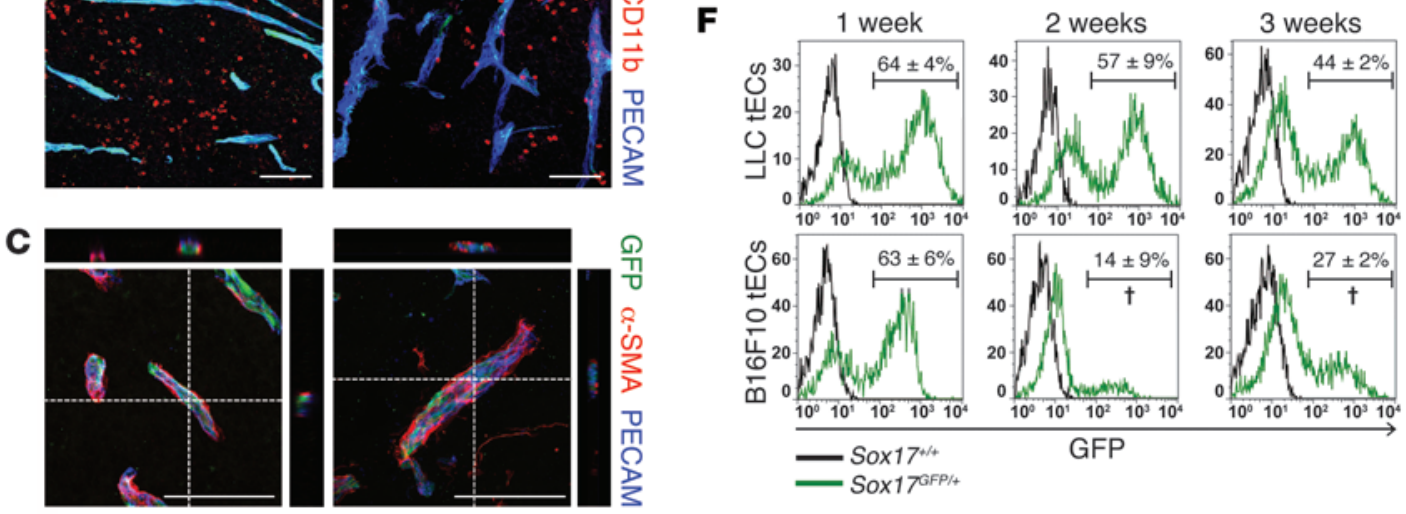

Sox $17^{4 / 4}$

GFP

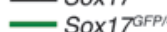

D

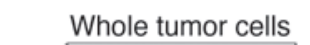

G
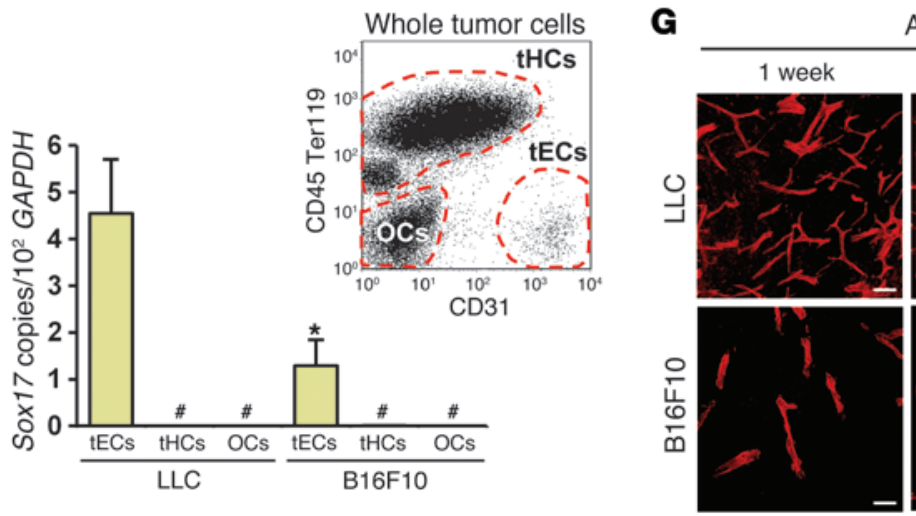

After implantation
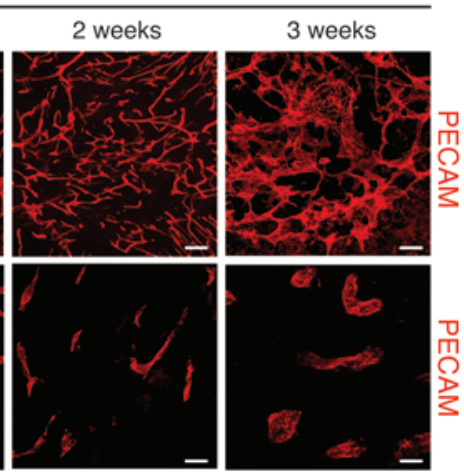

\section{Figure 1}

Sox17 expression specific to tECs is coincident with increased angiogenesis. LLC and B16F10 tumors grown in Sox17 GFP/+ mice (A-C and F) or wild-type mice (D, E, and G) were analyzed. (A-C) Images showing Sox17 expression (GFP) in tumor vessels (PECAM), but not in tumorassociated macrophages $\left(C D 11 b^{+}\right)$or pericytes $\left(\alpha-S M A^{+}\right), 2$ weeks after implantation. (D) Sox 17 transcripts specific to tECs purified from tumors 2 weeks after implantation. FACS plot shows cell populations purified from tumors. tHCs, tumor hematopoietic cells; OCs, other cells. (E) Sox17 expression in tECs at 1,2, and 3 weeks after implantation. (F) Sox17 expression in individual tECs, as determined by FACS. Percentages of Sox $17^{\text {high }}$ tECs are shown. Cells from Sox $17^{+/+}$mice were used to establish background expression. (G) Images of tumor vessels showing different patterns of angiogenesis in LLC and B16F10 tumors at 1, 2, and 3 weeks after implantation. (A-C and G) Scale bars: $100 \mu \mathrm{m}$. (D-F) $n=3$ (D and E) or 3-5 (F) per group. ${ }^{*} P<0.05,{ }^{\dagger} P<0.01$ versus LLC tECs; ${ }^{\dagger} P<0.001$ versus tECs.

endothelial compartment of tumor vessels. By analyzing the expression of Sox17 in tECs at the individual cell level, we showed that tECs were heterogeneous. Taken together, our findings demonstrated that transcriptional regulation by Sox 17 plays a critical role in tumor angiogenesis and in the morphogenesis of tumor vessels.

\section{Results}

Sox 17 is specifically expressed at high levels in tECs during tumor progres sion. Because Sox17 is expressed in blood vessels during development (24), we wondered whether it is also expressed in tumor ves- sels. 2 weeks after Lewis lung carcinoma (LLC) implantation, GFP tracing in Sox $17^{G F P /+}$ mice revealed that most of the tumor vessels expressed Sox17 at high levels in primary tumors (Figure 1A) and in pulmonary metastatic nodules (Supplemental Figure 1; supplemental material available online with this article; doi:10.1172/ JCI64547DS1). In implanted B16F10 melanomas, the tumor vessels also expressed Sox17, but at lower levels (Figure 1A). Sox17 expression was not detected in other stromal cells, including tumor-associated macrophages and pericytes, in both types of tumors (Figure 1, B and C). Quantitative RT-PCR analysis of purified cells from 
A
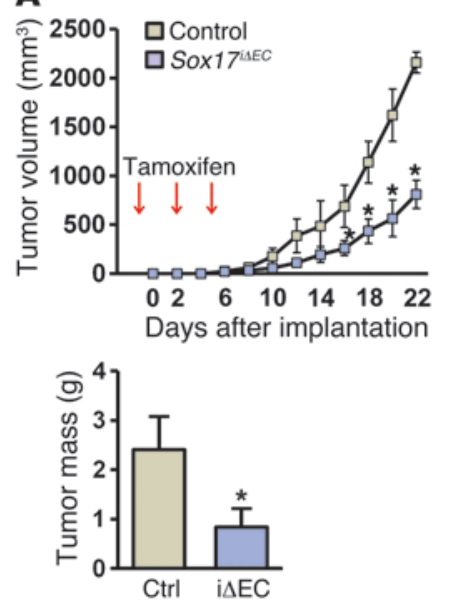

B

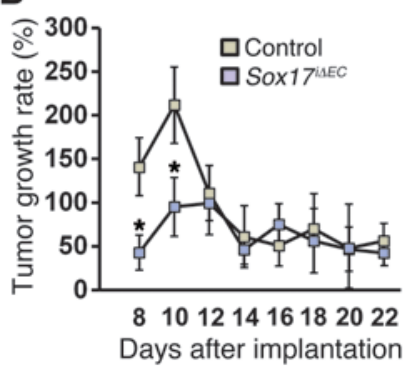

C
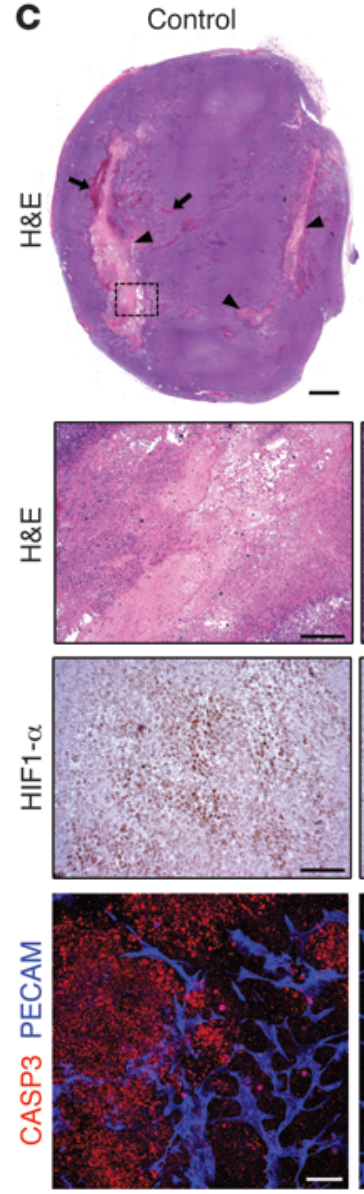

SOX17 $7^{\mathrm{AEC}}$
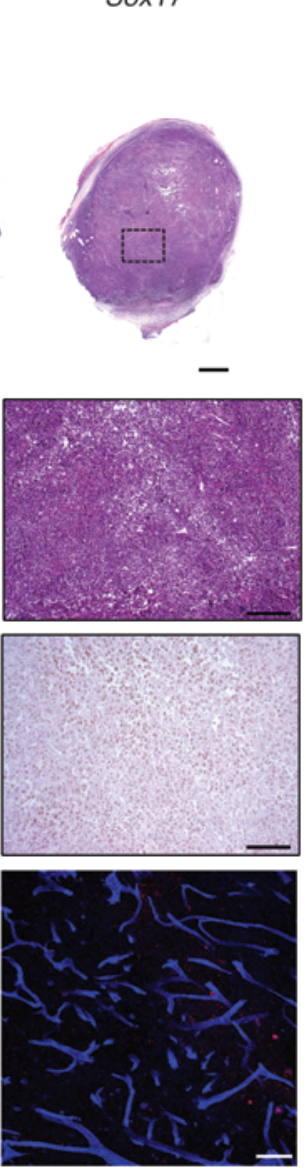

D Control
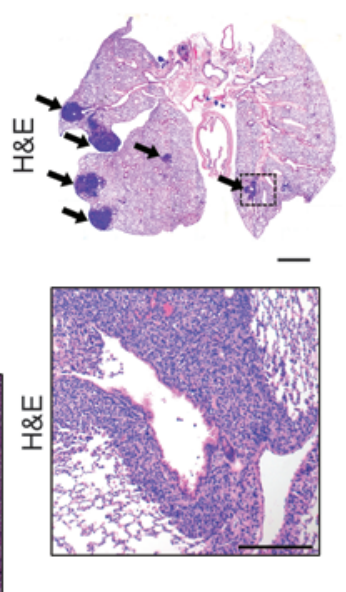

E

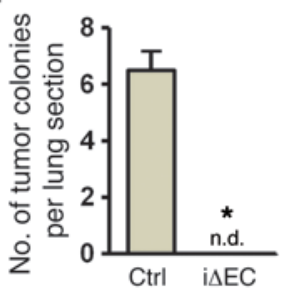

\section{Figure 2}

Sox17 deletion in tECs inhibits tumor progression, necrosis, and hypoxia. LLC tumors grown in control and Sox17isEC mice were analyzed. (A) Tumor growth (volume and mass) was inhibited in Sox17iAEC mice. Red arrows denote tamoxifen administration to elicit Sox17 deletion. (B) Tumor growth rates showed increased tumor volume relative to that 2 days earlier. (C) H\&E staining of tumor sections showed hemorrhage (arrows) and necrosis (arrowheads) in controls. Boxed regions are shown at higher magnification. HIF1- $\alpha$ staining and cleaved caspase-3 staining of tumor sections are also shown. (D) H\&E staining of lung sections showed clustered metastatic LLC tumors (arrows). Boxed regions are shown at higher magnification. (E) Quantitation of metastatic nodules $>200 \mu \mathrm{m}$ in diameter in mouse lungs. n.d., not detectable. (A, B, and E) $n=5-6(\mathbf{A}$ and B) or 3 (E) per group. * $P<0.01$ versus control. (C and D) Scale bars: $2 \mathrm{~mm}$ (C), top); $400 \mu \mathrm{m}$ (C, all others); $4 \mathrm{~mm}$ (D, top); $200 \mu \mathrm{m}$ (D, bottom).

tumors 2 weeks after implantation consistently detected Sox17 transcripts only in CD $31^{+} \mathrm{CD} 45^{-}$Ter119- $\mathrm{tECs}$ from both tumors. The transcript levels were approximately 4-fold higher in the LLC tumors than in the B16F10 tumors, which suggests that Sox17 expression may be regulated differentially depending on tumor type. Notably, there were negligible levels of Sox 17 transcripts in CD 31-CD45+Ter119+ tumor hematopoietic cells and in the other cell types within both tumor types (Figure 1D). These results indicate that Sox 17 expression within the tumors was specific to tECs.

We further examined Sox 17 expression in tECs over time during tumor progression. Sox17 expression in tECs gradually declined, but was detectable up to 3 weeks after implantation of both types of tumors. Sox 17 expression was approximately 2.5-fold higher in LLC tECs than in B16F10 tECs 2 and 3 weeks after implantation (Figure 1E). Flow cytometric analysis of Sox 17 expression also showed that tECs in LLC tumors expressed Sox17 at higher levels than tECs in B16F10 tumors 2 and 3 weeks after implantation (Figure 1F). Although abnormal tumor vessel features - such as enlargement, tortoise shell shape, and disorganized structure were common in both types of tumors during their progression, tumor vessel density and branching were considerably more pronounced in LLC tumors than in B16F10 tumors 2 and 3 weeks after implantation (Figure $1 \mathrm{G}$ ). In contrast to its strong expression in tECs, Sox 17 expression was barely detectable in most quiescent vessels in the postnatal pups and in the host, other than moderate expression in arterial vessels (Supplemental Figure 2, A and C). However, Sox17 expression was robust in angiogenic vessels, such as postnatal retinal vessels (Supplemental Figure 2B), which suggests that Sox17 expression is upregulated not only in tumor angiogenesis, but in other active angiogenesis contexts. To look for an upstream regulator of Sox 17 expression, we examined Sox 17 expression in primary ECs after treatment of established angiogenic stimuli. VEGF, FGF2, and hypoxia did not stimulate Sox17 expression, although they substantially activated the expression of known downstream genes (Supplemental Figure 3). The mechanisms underlying Sox17 expression in tumor angiogenesis remain 

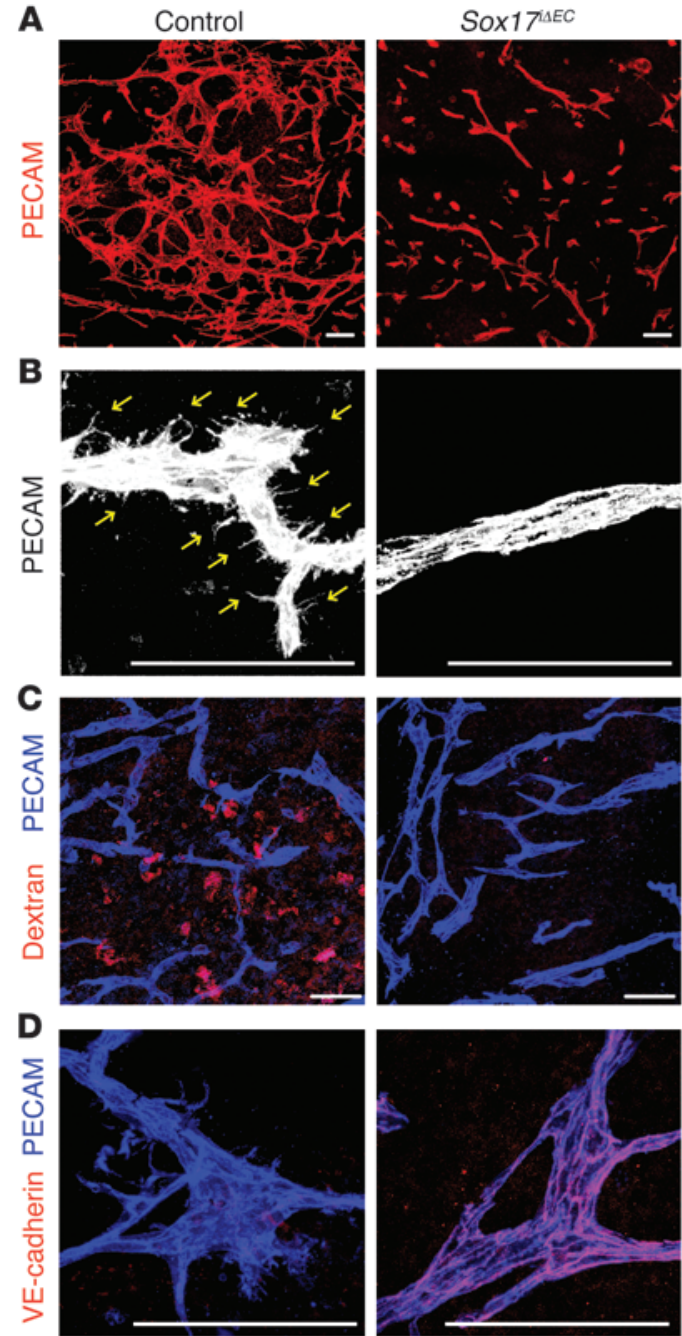

E
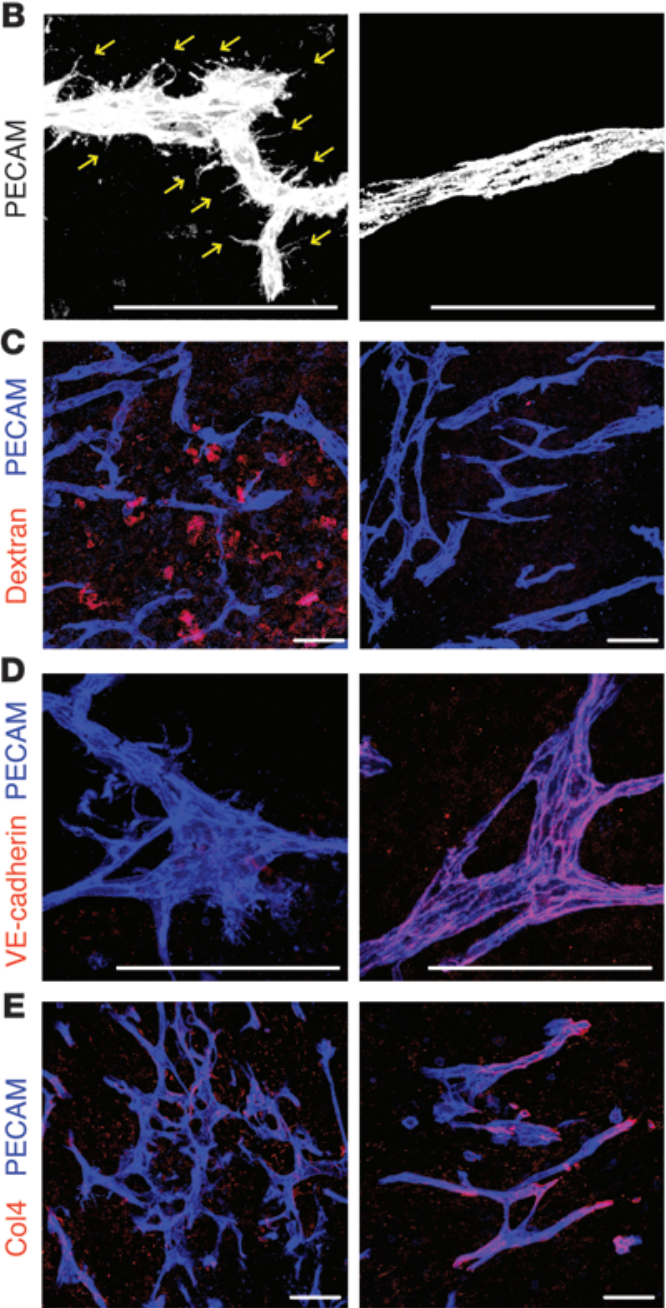

F
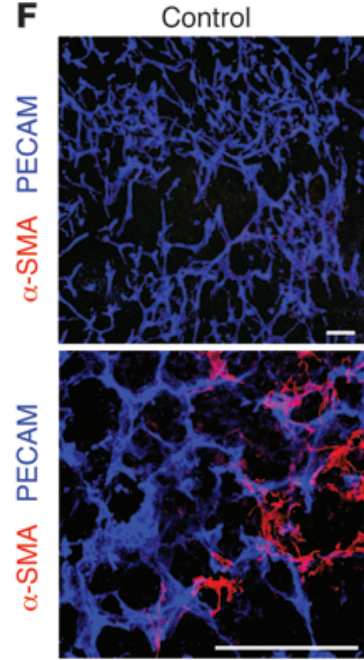

G

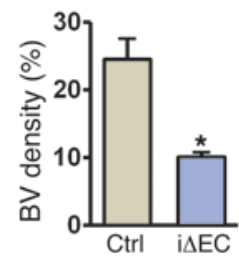

I

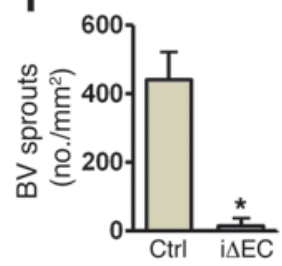

K

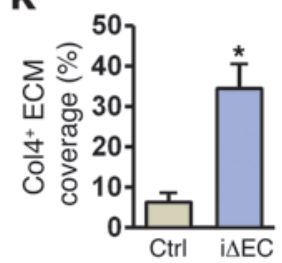

H

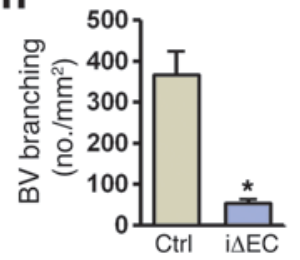

J

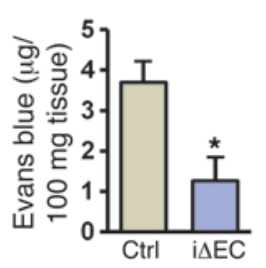

$\mathbf{L}$

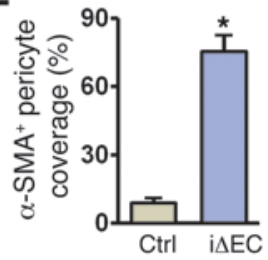

\section{Figure 3}

Sox17 deletion in tECs reduces tumor angiogenesis and vascular abnormalities. Tumor vessels were analyzed in LLC tumors grown in control and Sox17 1 iLEC mice. (A) Images of tumor vessels. (B) Filopodial protrusions, indicated by yellow arrows on the outside surfaces of tumor vessels. (C) Dextran release from the tumor vessels. (D) VE-cadherin staining of tumor vessels. (E) Col4 staining showing the basement membrane in tumor vessels. (F) $\alpha$-SMA staining showing pericytes in tumor vessels. Higher-magnification images are shown below to better visualize the association of pericytes and endothelium. ( $G$ and $\mathbf{H}$ ) Quantitation of blood vessel (BV) density and branches in tumors. (I) Quantitation of filopodial extensions on tumor vessels. (J) Quantitation of extravasated Evans blue in tumors. (K and $\mathbf{L}$ ) Quantitation of tumor vessels surrounded by Col4 matrix and $\alpha$-SMA+ pericytes. (A-F) Scale bars: $100 \mu \mathrm{m} .(\mathbf{G}-\mathbf{L}) n=3$ per group. ${ }^{*} P<0.01$ versus control.

unknown and merit further investigation. These data indicated that Sox17 expression in tECs is coincident with increased angiogenesis during tumor progression, which suggests that Sox17 may play a role in tumor angiogenesis.

Sox17 deletion in tECs inhibits tumor progression, tumor angiogenesis, and vascular abnormalities. To elucidate the role of Sox17 in
tECs during tumor angiogenesis, we generated a loss-of-function (LOF) mouse model to inducibly delete Sox17 in ECs [Cdh5(PAC)$\mathrm{CreER}^{T 2}$;Sox $17^{f / f l}$; referred to herein as Sox $\left.17^{i \Delta E C}\right]$. The Sox 17 allele was efficiently deleted in tECs of Sox $17^{i \triangle E C}$ mice upon tamoxifen administration (Supplemental Figure 4). When Sox17 was excised at the onset of tumor growth, Sox $17^{i \Delta E C}$ mice showed 
A

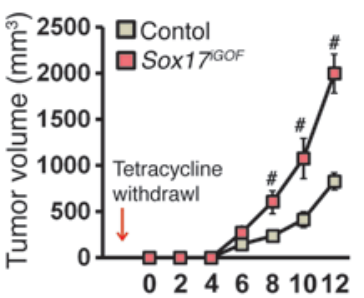

Days after implantation

C
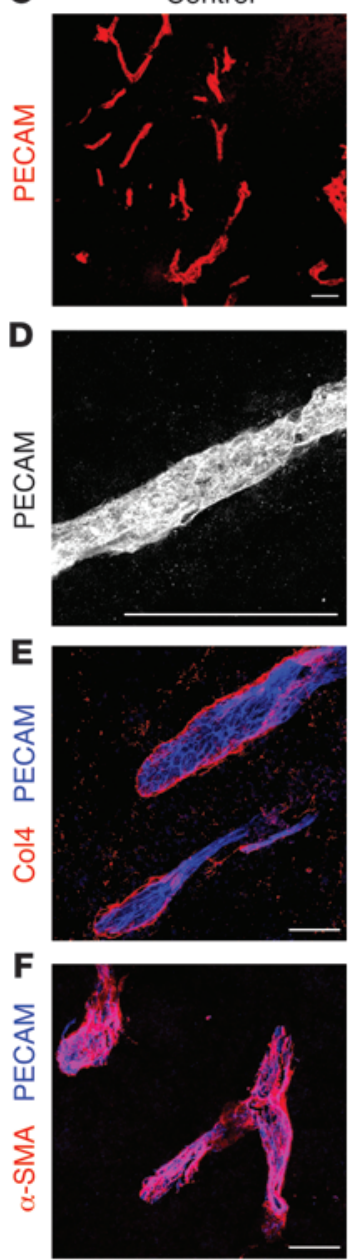

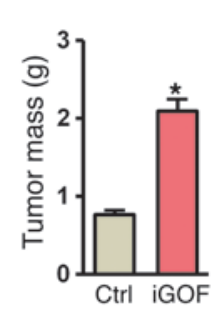

Sox17GOF
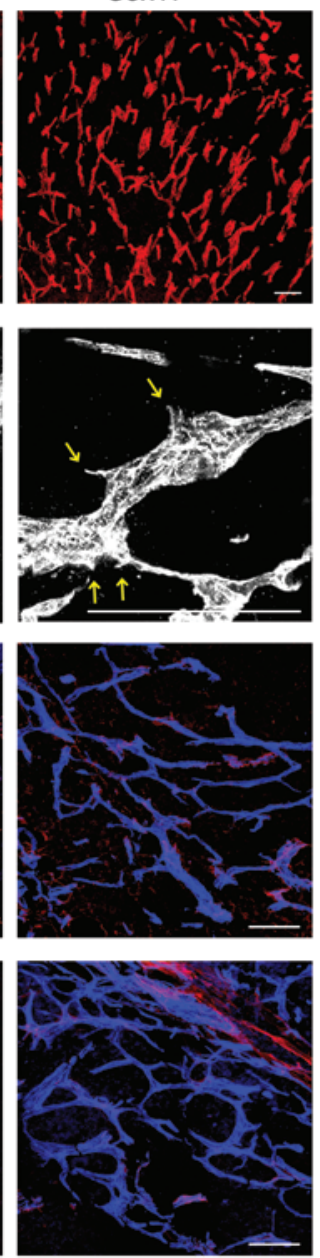

B

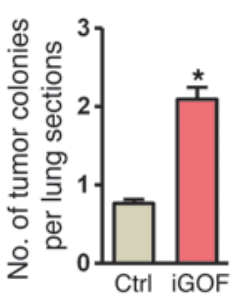

G
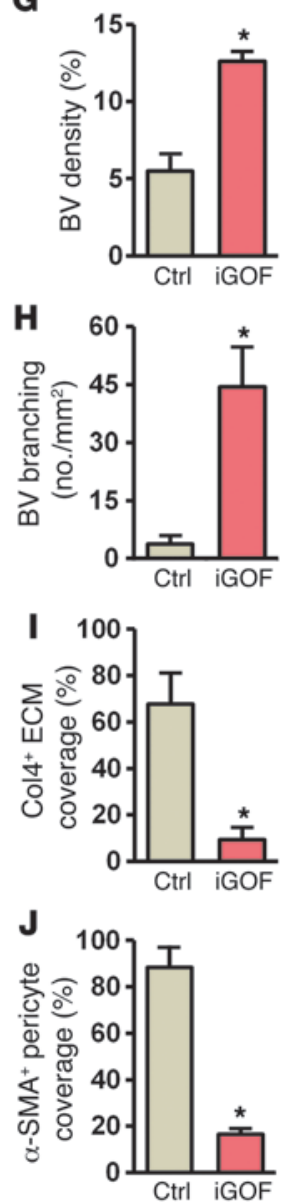

Figure 4

Gain of Sox17 in tECs promotes tumor progression, tumor angiogenesis, and vascular destabilization. B16F10 tumors grown in control and Sox17igof mice were examined. (A) Tumor growth (volume and mass). Tetracycline was withdrawn (red arrow) to allow for Sox17 overexpression. (B) Quantitation of metastatic nodules $>200 \mu \mathrm{m}$ in diameter in mouse lungs. (C) Images of tumor vessels showing increased tumor angiogenesis in Sox17iGOF mice. (D) Filopodial extensions (yellow arrows) on the outside surfaces of tumor vessels. (E and F) Col4 and $\alpha$-SMA staining of tumor vessels. ( $\mathbf{G}$ and $\mathbf{H}$ ) Quantitation of tumor vessel density and branches. (I and $\mathbf{J}$ ) Quantitation of tumor vessels surrounded by Col4 matrix and $\alpha-$ SMA $^{+}$pericytes. (A, B, and $\left.\mathbf{G}-\mathbf{J}\right) n=3(\mathbf{A}, \mathbf{B}, \mathbf{G}$, and $\mathbf{H}$ ) or 3-4 (I and $\mathbf{J}$ ) per group. ${ }^{\#} P<0.05,{ }^{*} P<0.01$ versus control. (C-F) Scale bars: $100 \mu \mathrm{m}$.

nodules 3 weeks after LLC tumor implantation (Figure 2, D and E). These results indicated that Sox17 stimulated tumor growth and metastasis by regulating tumor angiogenesis.

We next analyzed LLC tumor vessels 3 weeks after implantation. The vascular density in Sox $17^{i \Delta E C}$ mouse tumors was $60 \%$ lower than that in controls (Figure 3, A and G), while there was approximately $85 \%$ less vascular branching (Figure $3, \mathrm{~A}$ and $\mathrm{H}$ ) and approximately $65 \%$ less vascular leakage (Figure 3, C and J). Filopodial extensions were also remarkably reduced in Sox17-deficient compared with control tumor vessels (Figure 3, B and I). Notably, junctional VE-cadherin (Figure 3D), ECM collagen 4 (Col4; Figure $3, \mathrm{E}$ and $\mathrm{K}$ ), and $\alpha-\mathrm{SMA}^{+}$pericytes (Figure $3, \mathrm{~F}$ and $\mathrm{L}$ ) were retained well in Sox17-deficient versus control tumor vessels. Interestingly, the effect of Sox17 deletion on pericyte coverage in tumor vessels was specific to $\alpha$-SMA ${ }^{+}$pericytes, as nerve/glial antigen 2-positive (NG2-positive) pericytes showed no differences in terms of staining of control and Sox17deficient tumor vessels (Supplemental Figure 6). More studies are needed to elucidate the mechanisms underlying increased pericyte coverage in tumor vessels subsequent to Sox17 deletion. We further examined the effect of Sox 17 deletion on B16F10 tumor vessels. The inhibitory effect of Sox 17 deletion on tumor angiogenesis was similar in B16F10 tumor models (Supplemental Figure 5, C and G-H). Junc-

an approximate $60 \%$ reduction in tumor growth at 3 weeks after LLC implantation, compared with control Sox $17 f l f l$ mice lacking the Cre allele (Figure 2A). Inhibition of the tumor growth rate was especially noticeable during the early period of growth, up to 12 days after tumor implantation (Figure $2 \mathrm{~B})$. Growth of B16F10 tumors also declined by approximately $60 \%$ in Sox $17^{i \triangle E C}$ mice (Supplemental Figure 5, A and B). Histological analysis revealed that necrosis, hemorrhage, caspasemediated cell death, and hypoxia were reduced in tumors of Sox $17^{i \triangle E C}$ versus control mice (Figure 2C), which indicates that Sox17 deletion may improve vascular function in tumors.

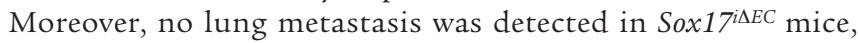
whereas most control mice had several pulmonary metastatic tional VE-cadherin distribution, coverage by $\alpha-\mathrm{SMA}^{+}$pericytes, and Col4 retention in B16F10 tumors did not show discernible increases in Sox17-deficient versus control tumor vessels (Supplemental Figure 5, D-F), which indicates that the effectiveness of Sox17 deletion varies in vascular structures depending on tumor type. These findings indicated that Sox17 deletion in tECs reduced tumor angiogenesis and improved vascular integrity.

Overexpression of Sox17 in tECs promotes tumor progression, tumor angiogenesis, and vascular abnormalities. To investigate whether elevated Sox 17 levels directly affect tumor vessels, we overexpressed Sox17 in tECs using a doxycycline-inducible gain-of-function (GOF) mouse model (VE-cadherin-tTA;Tet-O-Sox17; referred to herein as Sox17iGOF). Sox17 overexpression was observed in ECs 
A
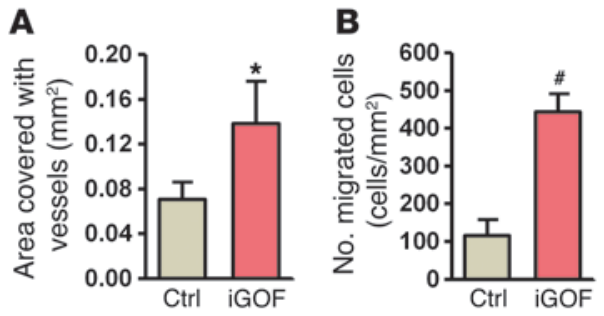

C
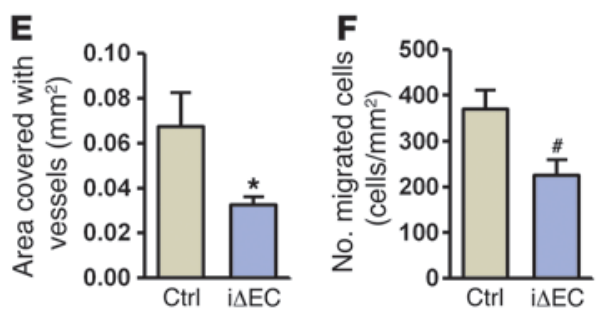

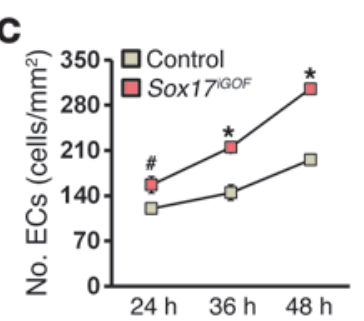

G

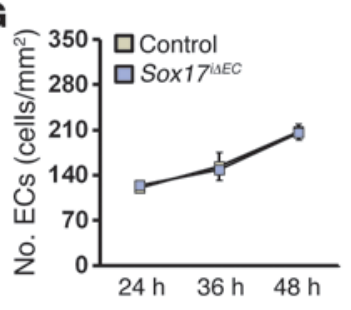

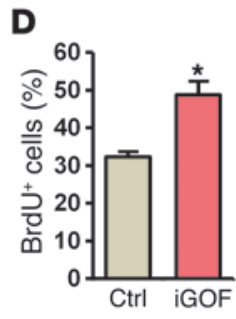

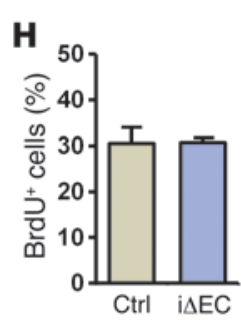

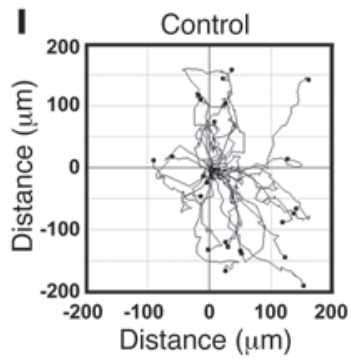

K

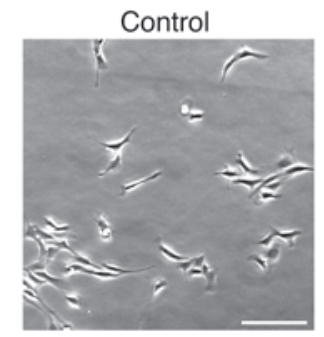

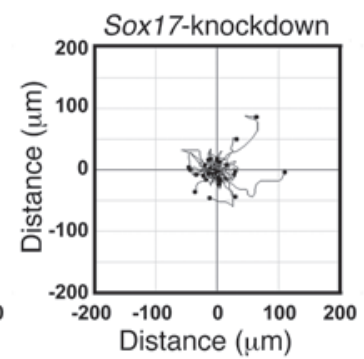

Sox17-knockdown

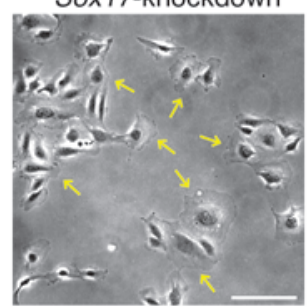

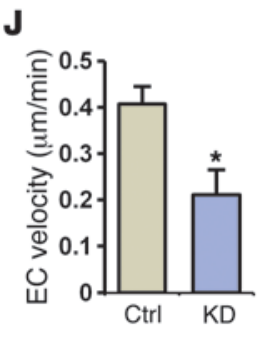

L

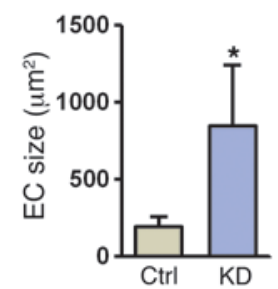

Figure 5

Sox17 promotes angiogenic behaviors of ECs. Aortic rings and lung ECs were obtained from genetic mouse models. (A-D) The effect of Sox17 overexpression was examined by removing doxycycline in cultures of cells from control and Sox17iGOF mice. (E-H) The effect of Sox17 deletion was studied by adding tamoxifen to cultures of cells from control and Sox17iAEC mice. (I-L) Control and Sox17-knockdown (KD) HUVECs were used. (A and $\mathbf{E}$ ) Sprout density in the aortic ring assay. (B and $\mathbf{F}$ ) Number of migrating cells in the wound scratch assay. ( $\mathbf{C}$ and $\mathbf{G}$ ) EC numbers increased during culture. ( $\mathbf{D}$ and $\mathbf{H}$ ) Percentage of BrdU-incorporated cells. (I) Trajectories of individual EC locomotion. The migration of individual ECs was captured using time-lapse microscopy and converted to a 2-dimensional graph. (J) Slow EC migration was observed upon Sox17 knockdown. (K) Whereas control ECs showed a spindle shape, Sox17knockdown ECs exhibited a spreading shape (yellow arrows). (L) Sox17 knockdown increased the area of EC surface adherent on the matrix. (A-H, J, and $\mathbf{L})$ $n=3(\mathbf{A}-\mathbf{H}), 20-23(\mathbf{J})$, or 32-34 (L) per group. ${ }^{*} P<0.05$, ${ }^{*} P<0.01$ versus control. (K) Scale bars: $100 \mu \mathrm{m}$. upon doxycycline withdrawal (Supplemental Figure 7). Although Sox 17 overexpression promoted modest tumor growth and tumor angiogenesis in LLC tumors compared with control VE-cadherin$t T A$ mice, there were no appreciable differences in vascular branching and structure (data not shown), which is not surprising, given that LLC tumors already express high levels of endogenous Sox17 and elicit a vigorous angiogenic response. We next examined the effect of Sox 17 overexpression in implanted B16F10 tumors, which in control mice showed weak Sox17 expression in the tumor vessels. Tumor growth and metastasis increased in Sox 17 iGOF relative to control mice 12 days after B16F10 implantation (Figure

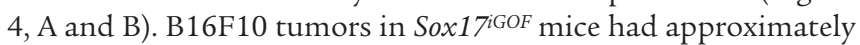
2.5-fold higher vascular density and approximately 11.6-fold more vascular branching than did control mice (Figure 4, C, G, and H). There were also more filopodial extensions in the tumor vessels of Sox $17^{i G O F}$ mice (Figure 4D). ECM Col4 and $\alpha$-SMA ${ }^{+}$pericytes were barely detectable in Sox 17-overexpressing compared with control B16F10 tumor vessels (Figure 4, E, F, I, and J). Together with the LOF studies described above, these GOF findings demonstrated that Sox17 in tECs had proangiogenic and destabilizing effects on tumor vessels that promote tumor progression.
Sox17 increases the angiogenic behaviors of ECs. Knowing that Sox17 promoted hyperbranching of tumor vessels, we further characterized the effect of Sox17 on EC behavior in vitro using cells and tissues from Sox 17 genetic mouse models. An aortic ring-based sprouting assay revealed that sprouting was increased approximately 2 -fold in Sox $17^{i G O F}$ compared with control aortic rings (Figure 5A and Supplemental Figure 8A). Conversely, sprouting was reduced approximately 2 -fold in Sox $17^{i \Delta E C}$ aortic rings compared with controls (Figure 5E and Supplemental Figure 8D). We next assessed endothelial migration and proliferation in primary ECs derived from Sox17 genetic mouse models. In the wound scratch cell migration assay, migration increased approximately 4-fold in Sox $17^{\mathrm{IGOF}}$ ECs and decreased approximately 1.6-fold in Sox $17^{\mathrm{i} A E C}$ ECs compared with the appropriate controls (Figure 5, B and F, and Supplemental Figure 8, B and E). ECs from Sox17iGOF mice were more proliferative than control ECs (Figure 5, C and D, and Supplemental Figure 8C), whereas there was no difference in the proliferative activity of ECs from control and Sox $17^{i \triangle E C}$ mice (Figure 5, G and H, and Supplemental Figure 8F). This indicates that regulation of mitotic behavior by Sox17 does not depend solely on Sox17 levels. Time-lapse tracking showed that Sox17-knockdown 

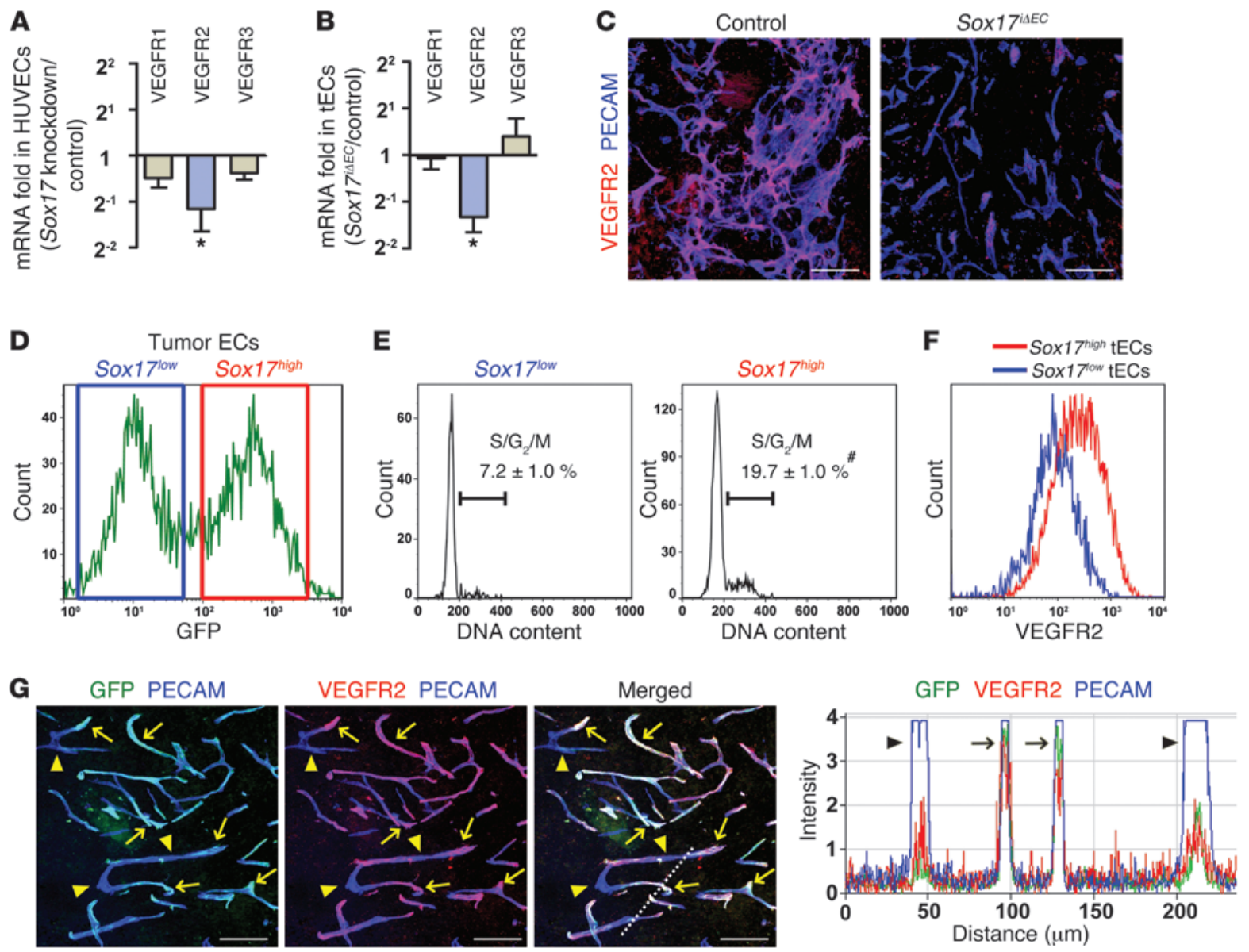

\section{Figure 6}

Sox17 increases VEGFR2 expression in tECs. (A and B) Transcript level of VEGFRs in (A) Sox17-knockdown HUVECs and (B) tECs from LLC tumors in Sox17iLEC mice and respective controls. Levels were normalized based on GAPDH mRNA levels and are shown as fold difference compared with control. (C) VEGFR2 staining in LLC tumor vessels in control and Sox17iLEC mice. (D) FACS plot showing 2 distinct subpopulations of tECs from LLC tumors grown in Sox $17^{\mathrm{GFP} /+}$ mice. Sox17 high (red) and Sox17low (blue) tECs were fractionated for subsequent analyses. (E) DNA content measured in individual tECs to determine cell cycle status. The percentage of cells in the $S / G_{2} / M$ phases of the cell cycle is indicated. (F) FACS plot showing VEGFR2 expression on the surfaces of tECs, indicative of VEGFR2 upregulation on Sox17 high tECs. Data from 2 histograms from Sox $17^{\text {high }}$ and Sox17low tECs were merged. (G) Coincident expression of Sox17 (GFP) and VEGFR2 in LLC tumors grown in Sox17GFP/+ mice. Arrows denote areas of tumor vessels with high expression of both Sox17 and VEGFR2. Arrowheads indicate areas with weak expression of both Sox17 and VEGFR2. The histogram shows the intensities of GFP, VEGFR2, and PECAM expression at each pixel along the dotted line shown in the merged image. (A, B, and $\mathbf{E}) n=3$ per group. ${ }^{*} P<0.05$ versus control; ${ }^{\#} P<0.01$ versus Sox 17 low. $(\mathbf{C}$ and $\mathbf{G})$ Scale bars: $100 \mu \mathrm{m}$.

HUVECs had reduced motility - as demonstrated by an approximate 2 -fold decrease in velocity - and a more localized roaming pattern compared with controls (Figure 5, I and J). In addition, control HUVECs elongated rapidly upon VEGF stimulation, whereas Sox17-knockdown HUVECs remained flattened, as shown by the approximately 4-fold greater spreading area of Sox17-knockdown HUVECs compared with control cells (Figure 5, K and L). This indicated that Sox 17 deficiency in ECs results in an impaired shape change in response to a stimulus that promotes migration, such as VEGF. Thus, Sox17 promoted sprouting angiogenesis by stimulating EC mitosis and migration in a cell-autonomous manner.

Sox 17 upregulates VEGFR2 expression in $t E C$. To investigate the molecular mechanism by which Sox17 controls EC behavior, we compared gene expression in control and Sox17-knockdown HUVECs using microarrays. Of about 28,000 genes, Sox 17 knockdown upregulated 53 genes and downregulated 32 genes at least 2.5-fold (Supplemental Tables 1 and 2). The genes most downregulated by Sox 17 knockdown were the chemokines CXCR7 and CCL2, which are involved in immune cell chemoattraction (27). Among the genes involved in angiogenic regulation, only ephrin-B2 (downregulated 3.5-fold) and VEGFR2 (downregulated 3.2 -fold) showed significant changes in response to Sox 17 knockdown. Sox17 knockdown also altered the expression of some genes encoding signaling molecules, ECM components or related proteins involved in ECM posttranslational modification, and secretory molecules or related proteins for their exocytosis. These results indicated that Sox17 regulates the expression of a small set of genes in ECs for its angiogenic function.

Because VEGFR2 has a fundamental role in tumor angiogenesis, we examined VEGFR2 expression in Sox17-knockdown ECs and Sox17 genetic mouse models using quantitative RT-PCR. VEGFR2 transcripts were decreased more than 2-fold in primary Sox17- 
A
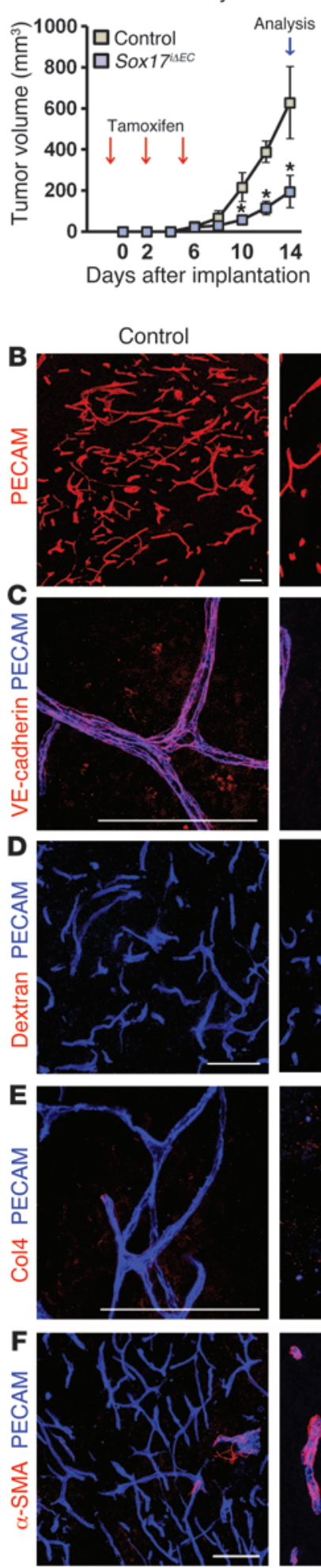
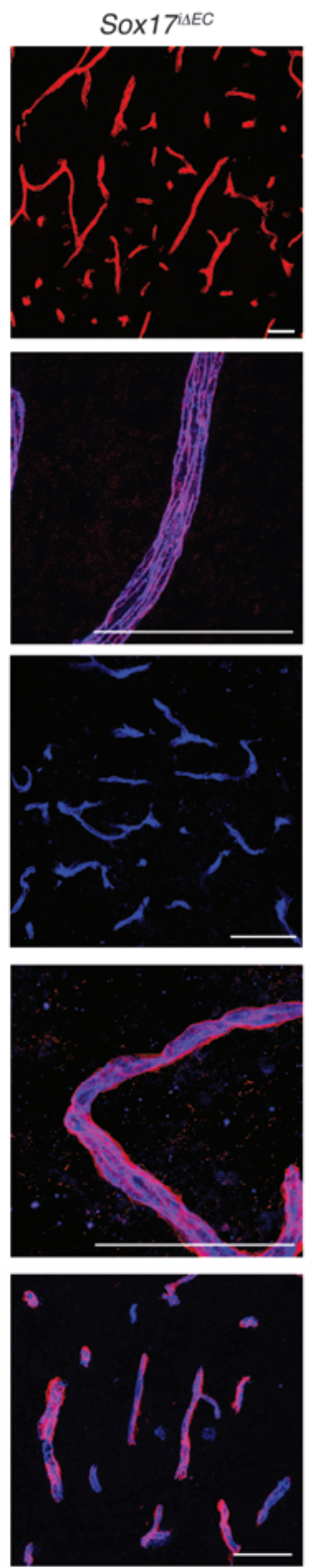

G
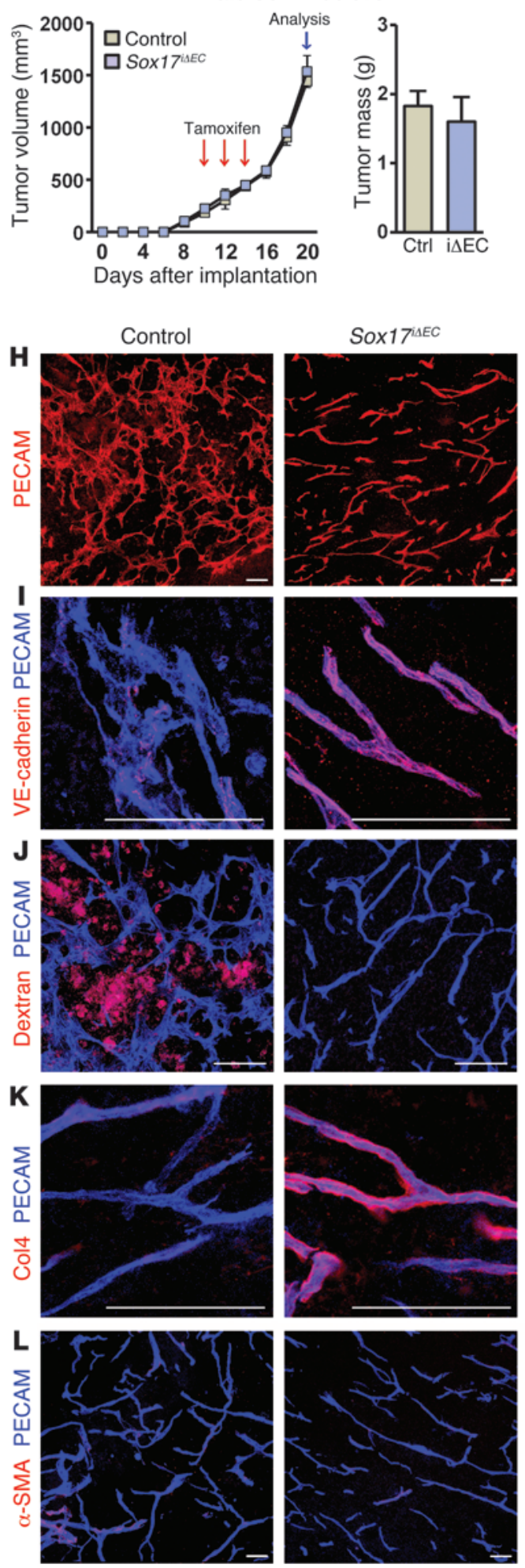

Figure 7

Sox17 deletion in tECs persistently inhibits tumor angiogenesis and vascular abnormalities. Tumors were grown in control and Sox17iLEC mice, and tamoxifen was administered (red arrows) for early $(\mathbf{A}-\mathbf{F})$ or late $(\mathbf{G}-\mathbf{L})$ Sox17 deletion. (A and $\mathbf{G})$ Tumor growth (volume and mass). Blue arrows denote the time point of tumor analysis. (B and $\mathbf{H}$ ) Tumor angiogenesis was reduced in Sox17iLEC tumors. (C and I) VE-cadherin staining in tumor vessels. (D and $\mathbf{J})$ Extravasated dextran, indicative of vascular leakage. (E and $\mathbf{K})$ Col4 staining of the basement membrane in tumor vessels. $(\mathbf{F}$ and $\mathbf{L}) \alpha$-SMA staining of the pericytes in tumor vessels. $(\mathbf{A}$ and $\mathbf{G}) n=4-5$ per group. ${ }^{*} P<0.01$ versus control. (B-F and $\left.\mathbf{H}-\mathbf{L}\right)$ Scale bars: $100 \mu \mathrm{m}$. For quantitative analysis of B, E, F, H, K, and L, see Supplemental Figure 10. 
A
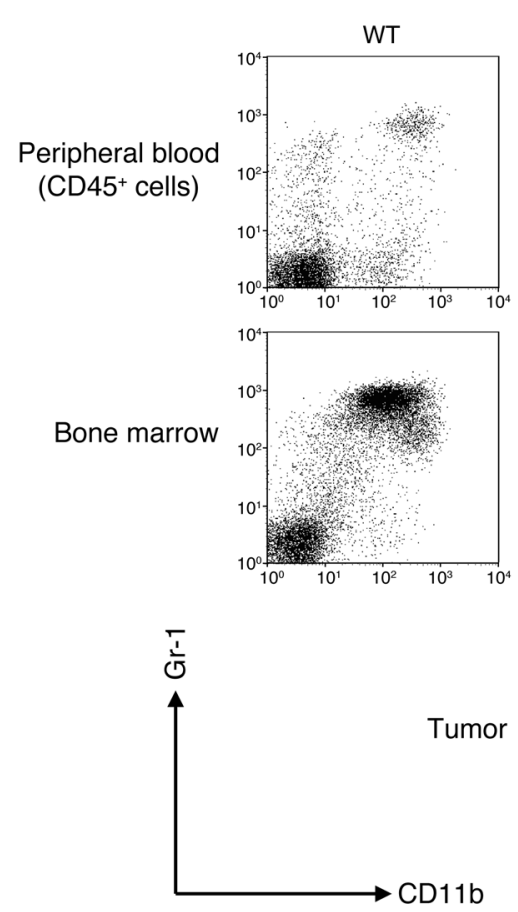

Early Sox 17 deletion
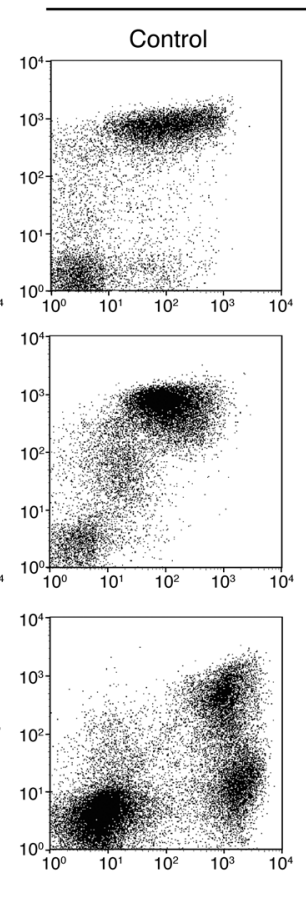
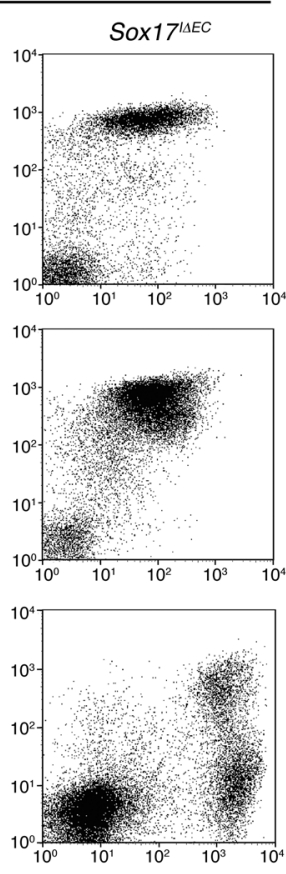

Late Sox17 deletion
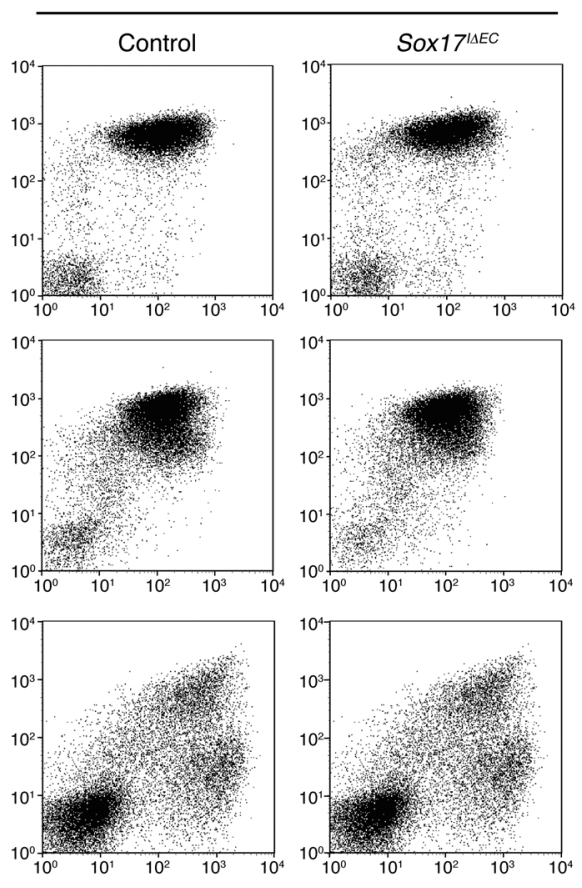

B

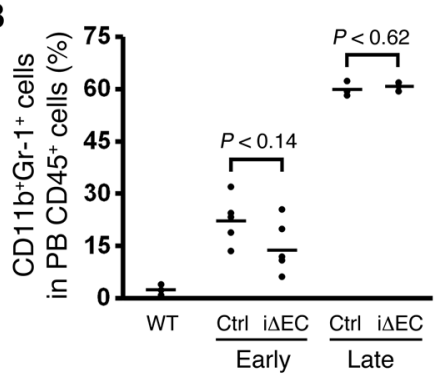

C

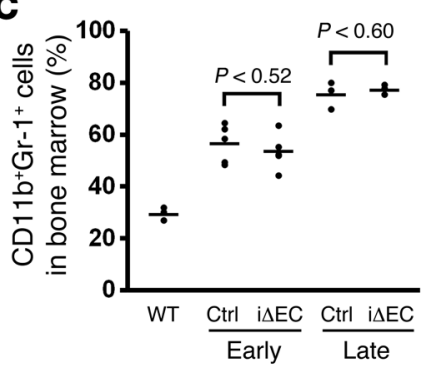

D

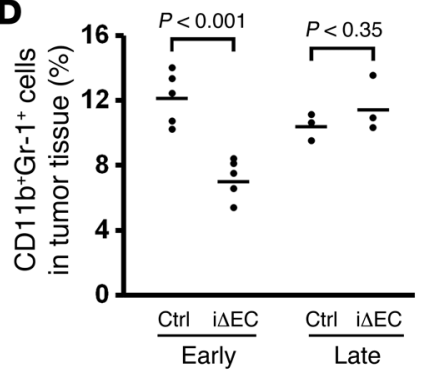

Figure 8

Sox17 promotes recruitment of inflammatory cells into tumors. Tumors were grown in control and Sox $17^{i \Delta E C}$ mice, and tamoxifen was administered for early or late Sox17 deletion. (A) FACS plots showing CD11b+Gr-1+ cells in peripheral blood, bone marrow, and tumors. (B-D) Percent CD11b+Gr-1+ cells in peripheral blood (PB; B), bone marrow (C), and tumor tissues (D). Peripheral blood cells were gated by $\mathrm{CD}_{4} 5^{+} . n=3-5$ per group.

knockdown ECs compared with controls (Figure 6A), confirming the microarray results and suggesting an intrinsic role of Sox17 in VEGFR2 expression. We consistently found reduced levels of both VEGFR2 mRNA (Figure 6B) and VEGFR2 protein (Figure 6C) in Sox $17^{i \triangle E C}$ compared with control tECs. Thus, Sox 17 promotes VEGFR2 expression in tECs. These results suggested that regulation of VEGFR2 expression could be one of the molecular mechanisms underlying Sox17-mediated tumor angiogenesis.

Different tEC populations show distinct Sox 17 expression levels. Because the tECs were heterogeneous with regard to Sox17 expression level, we characterized 2 tEC populations, Sox17high and Sox17low, at the individual cell level using flow cytometry. These populations were fractionated from LLC tumors grown in Sox $17^{\mathrm{GFP} /{ }^{+}}$mice (Figure $6 \mathrm{D})$. Whereas $19.7 \% \pm 1.0 \%$ of Sox $17^{\text {high }} \mathrm{tECs}$ were in the $\mathrm{S} / \mathrm{G}_{2} / \mathrm{M}$ phases of the cell cycle, only $7.2 \% \pm 0.7 \%$ of Sox $17^{\text {low }}$ tECs were in the $S / G_{2} / M$ phases (Figure $6 \mathrm{E}$ ), indicative of a functional difference between these tEC populations. The mean fluorescent intensities of VEGFR2 on individual cells were $579 \pm 42(n=3)$ in Sox17high tECs and $350 \pm 35(n=3)$ in Sox17 low tECs (Figure 6F). Accordingly, VEGFR2 expression paralleled Sox 17 expression in tumor vessels (Figure 6G). These findings indicated that in a heterogeneous cell population, high Sox17 expression distinguishes tECs that divide more rapidly and have higher levels of surface VEGFR2.

The effect of Sox 17 on tumor angiogenesis and vascular abnormality persists throughout the growth of the tumor. To investigate whether Sox17 has a proangiogenic effect throughout tumor growth, we deleted Sox17 at different time points. To examine the effect of Sox17 in early stages of tumor growth, Sox 17 was deleted at the onset of tumor growth (referred to herein as early Sox17 deletion), and tumors were analyzed 2 weeks after LLC implantation. To examine the effect of late deletion, Sox17 was deleted 10 days after tumor implantation (late Sox17 deletion), and tumors were examined 3 weeks after LLC inoculation. Early Sox17 deletion significantly delayed tumor growth, whereas late Sox17 deletion did not appear 


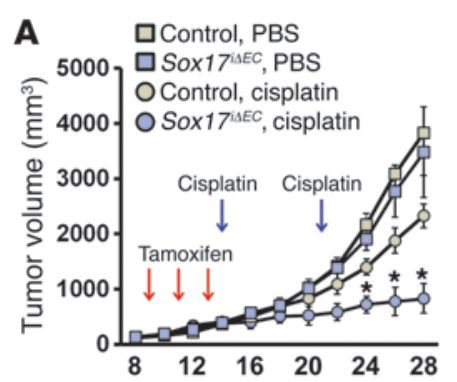

Days after tumor implantation

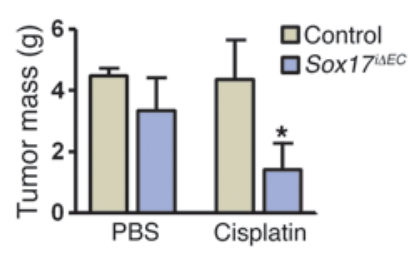

C

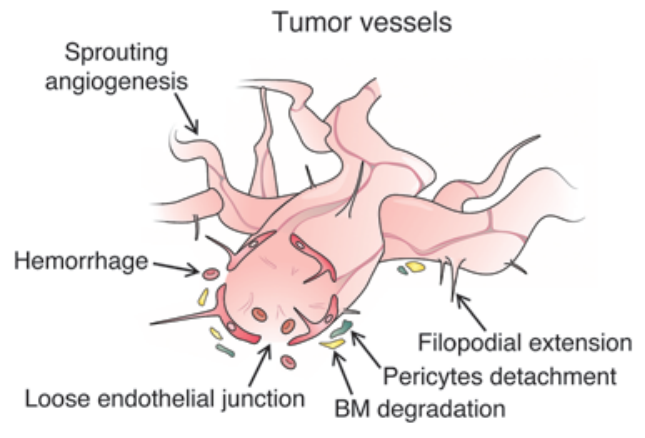

B Control, PBS

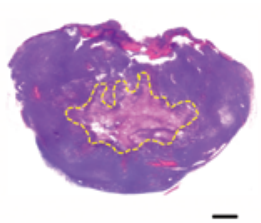

Control, cisplatin

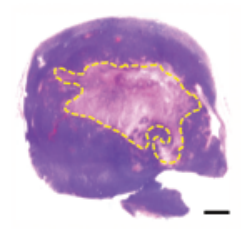

SOX17iAEC, PBS

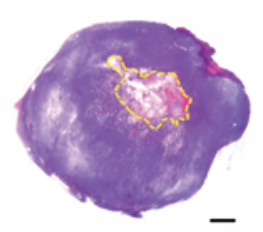

Sox17 ${ }^{\mathrm{ISEC}}$, cisplatin

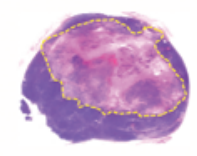

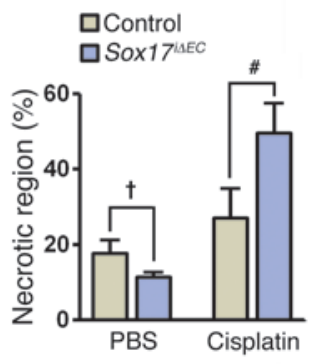

Tumor vessels

in Sox17 deletion

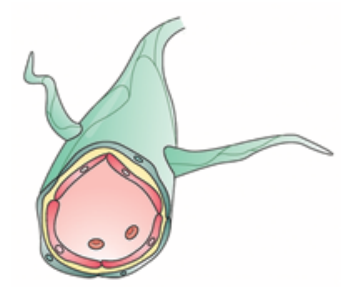

Normalization

Less angiogenesis

\section{Figure 9}

Late Sox17 deletion inhibits tumor growth by improving the efficacy of chemotherapy. Tumors were grown in control and Sox $17^{i \Delta E C}$ mice. (A) Tumor growth (volume and mass). Red arrows indicate tamoxifen administration for delayed Sox17 deletion. Cisplatin (or PBS as vehicle) was administered 2 and 3 weeks after implantation (blue arrows). (B) H\&E staining, showing tumor necrotic regions (dashed yellow outlines), and necrosis quantitation. (C) Effect of Sox17 deletion on tumor vessel morphogenesis. Tumor vessels (left) are characterized by excessive angiogenesis and poor integrity. Sox17 deletion in tECs (right) inhibits tumor angiogenesis and induces tumor vessel normalization. ECs are shown in red; pericytes are shown in green; basement membrane (BM) is shown in yellow. (A and $\mathbf{B}) n=5$ per group. ${ }^{*} P<0.05$ versus all other groups; t $P<0.05 ; \# P<0.01$. Scale bars: $4 \mathrm{~mm}$.

to have an effect (Figure 7, A and G). Along with our finding that the tumor growth rate was inhibited by Sox17 excision only during the early period (Figure $2 \mathrm{~B}$ ), these data indicated that in tECs, Sox 17 promotes tumor growth mainly during the early period of tumor angiogenesis. Although lung metastasis was not detectable 2 weeks after tumor implantation either in control or early Sox17 deletion animals (data not shown), the number of pulmonary metastatic nodules was significantly reduced by late Sox17 deletion (Supplemental Figure 9, A and B). These data indicated that Sox17 continuously promotes tumor progression during the late period of tumor angiogenesis by increasing tumor metastasis.

Both early and late Sox17 deletion reduced tumor vascular density and branching compared with control mice (Figure 7, B and $\mathrm{H}$, and Supplemental Figure 10, A, B, E, and F). There were no differences in VE-cadherin distribution at endothelial junctions and in vascular leakage between control and early Sox 17 deletion tumors (Figure 7, C and D). However, in tumors with late Sox17 deletion, VE-cadherin distribution at endothelial junctions appeared normal and vascular leakage was not detected, whereas junctional
VE-cadherin distribution was markedly reduced and vascular leakage was abundant in control mice (Figure 7, I and J). In contrast to severe loss in control mice, Col4 was abundantly distributed around tumor vessels in both early and late Sox17 deletion tumors (Figure 7, E and K, and Supplemental Figure 10, C and G). In addition, $\alpha-\mathrm{SMA}^{+}$pericytes were prevalent in the vessels of early Sox 17 deletion tumors, in contrast to control tumor vessels (Figure 7F and Supplemental Figure 10D). In contrast, tumors from late Sox17 deletion mice lacked $\alpha-\mathrm{SMA}^{+}$pericytes, similar to controls (Figure 7L and Supplemental Figure 10H). Notably, tumor vessels were well-covered by pericytes initially and become denuded later in control mice (Supplemental Figure 11). Thus, improved pericyte coverage by early Sox 17 deletion and poor pericyte coverage by late Sox 17 deletion in tumor vessels might be interpreted as evidence that Sox17 deletion helps maintain, rather than recruit, pericytes. These results indicated that in tECs, Sox17 promotes persistent tumor angiogenesis and progressively disrupts vascular integrity from the periendothelial compartment into the endothelial layer. 

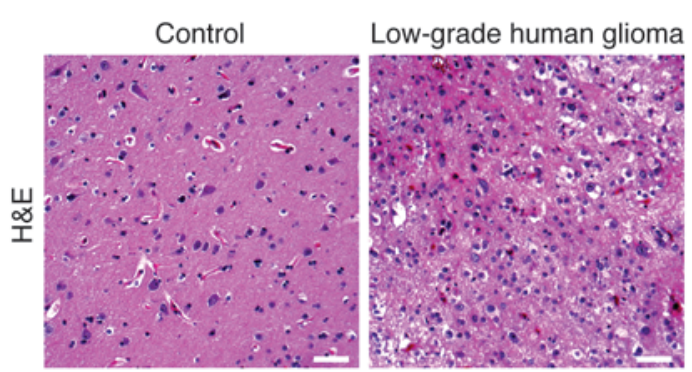

High-grade human glioblastoma

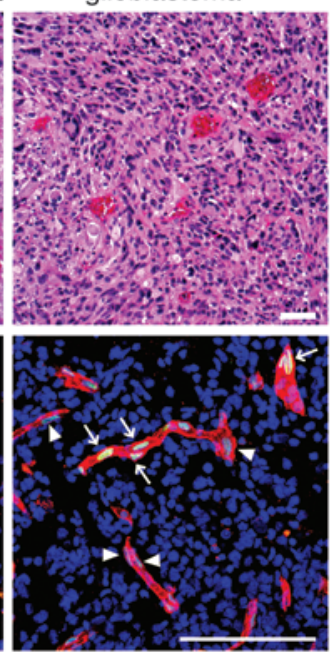

\section{Figure 10}

Sox17 is highly expressed in tumor vessels in human glioblastoma. Sox17 expression was analyzed in tissues from nontumor control human brain, low-grade human glioma, and high-grade human glioblastoma. H\&E staining shows cancer grade. Sox17 immunostaining shows nuclear Sox17 in tECs of high-grade glioblastoma (arrows and arrowheads denote strong and weak Sox17 expression, respectively). $n=3$ (control and low-grade human glioma) or 5 (high-grade human glioblastoma). Scale bars: $100 \mu \mathrm{m}$.
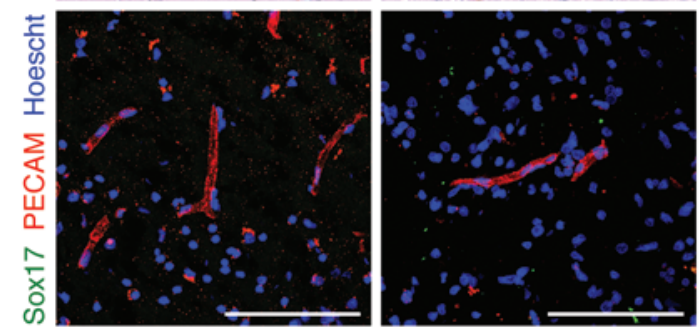

Sox17 promotes the recruitment of inflammatory cells into tumors. Given that the chemokine genes CXCR7 and CCL2 were some of the most downregulated genes after Sox 17 knockdown, based on our microarray data, we next sought to determine whether Sox17 deletion affects the recruitment of inflammatory cells into tumors. As $\mathrm{CD} 11 \mathrm{~b}^{+} \mathrm{Gr}-1^{+}$inflammatory cells recruited to LLC tumors promote tumor angiogenesis $(28,29)$, we examined the percentage of CD $11 b^{+} \mathrm{Gr}-1^{+}$myeloid cells after early or late Sox 17 deletion. Early Sox 17 deletion decreased the percentage of $\mathrm{CD} 11 \mathrm{~b}^{+} \mathrm{Gr}-1^{+}$cells by approximately $43 \%$ in LLC tumors, although the percentage was not altered significantly in peripheral blood and bone marrow (Figure 8, A-D). However, late Sox17 deletion had little effect on the percentage of $\mathrm{CD} 11 \mathrm{~b}^{+} \mathrm{Gr}-1^{+}$cells in peripheral blood, bone marrow, or tumor (Figure 8, A-D). These data indicated that Sox17 promotes the recruitment of $\mathrm{CD} 11 \mathrm{~b}^{+} \mathrm{Gr}-1^{+}$cells into tumors, rather than promoting cell mobilization and proliferation within bone marrow, during early tumor growth. This suggests, in turn, that recruitment of inflammatory cells into tumors is one of the mechanisms for Sox17-mediated tumor growth and tumor angiogenesis.

Sox17 deletion improves the efficacy of chemotherapy in the late tumor growth period. We hypothesized that because Sox17 deletion improved vascular integrity of the tumor, the vessels in late Sox17 deletion tumors might be able to effectively deliver anticancer drugs. This prompted us to examine whether cisplatin could reduce tumor growth in late Sox17 deletion tumors during the late period of tumor growth. Cisplatin, administered 2 and 3 weeks after LLC implantation, significantly reduced the growth of late Sox 17 deletion tumors by $70 \%$ compared with tumors in cisplatintreated controls, whereas PBS vehicle treatment did not make a discernible difference in tumor growth (Figure 9A). Histological analysis of tumor sections 4 weeks after implantation revealed that the combination of late Sox17 deletion and cisplatin treatment increased the necrotic region more than 2-fold in Sox $17^{i \Delta E C}$ compared with control mice (Figure 9B). In contrast, tumors in Sox $17^{i \Delta E C}$ mice had approximately 2 -fold less necrosis than did control tumors in the absence of cisplatin treatment (Figure 9B), consistent with reduced necrosis by early Sox 17 deletion (Figure 2C). Notably, the density and branching of tumor vessels were not visibly affected by cisplatin (data not shown), which excluded the possibility that cisplatin had a direct effect on tumor vessels. Together with the reduced vascular leakage observed as a result of late Sox17 deletion (Figure 7J), these findings revealed that even though Sox17 deletion had little effect on tumor growth during the late period, late Sox 17 deletion in tECs improved the efficacy of chemotherapy via effective drug delivery.

Sox 17 expression is robust in human glioblastoma tECs. To investigate the relevance of Sox 17 in human tumor angiogenesis, we examined Sox17 expression in human tumor vessels by immunostaining brain tumor sections, including glioblastoma, which is known to be highly vascularized (30). Nuclear Sox17 was detected predominantly and specifically in tECs in 4 of 5 high-grade human glioblastoma tissue samples, but Sox17 expression was not detectable in the 3 control brain tissues or 3 low-grade human glioma specimens examined (Figure 10). Considering the high vascularity of glioblastoma, these findings suggested that Sox17 expression in tECs is related to the angiogenic properties of tumor vessels. Interestingly, Sox17 expression in tECs was heterogeneous in human glioblastoma tissues, which indicates that the pattern of Sox17 expression in tumor angiogenesis was conserved in humans and mice. These findings are suggestive of the clinical relevance of Sox17 expression in tumor angiogenesis in some human tumors.

\section{Discussion}

Here we report that the transcription factor Sox17 expressed in tECs promoted tumor angiogenesis and vascular destabilization via upregulation of a key angiogenic receptor, VEGFR2, and enhanced recruitment of CD $11 \mathrm{~b}^{+} \mathrm{Gr}-1^{+}$cells into tumors (Figure 9C). Sox17 deletion inhibited tumor growth by reducing tumor angiogenesis and enhanced drug delivery via normalization of tumor vessels. Our findings highlight the importance of Sox17 in tumor angiogenesis and tumor progression.

In this study, Sox17 expression in ECs correlated with angiogenesis. Robust Sox17 expression in tECs contrasted with its weak expression in most quiescent vessels in adult hosts. Moreover, Sox17 expression was higher in tECs from tumors with hyperbranched and hyperdense vessels. The upstream regulation of Sox17 expression in tumor angiogenesis needs to be clarified in the future. Together with a previous report on Sox17 expression 
specific to ECs during vascular development (24), the present study demonstrated that Sox 17 faithfully follows the endothelialspecific expression in various angiogenic contexts, including tumor angiogenesis. Our characterization of individual tECs revealed 2 cell populations that were distinguished by their Sox17 expression, demonstrating the heterogeneity of tECs. Endothelial heterogeneity is observed in some circumstances during vascular development, for instance, during lineage specification into arterial, venous, and lymphatic ECs (31) and during the determination of tip and stalk cells (16). Phalanx ECs in prolyl hydroxylase domain-containing protein 2-insufficient (PHD2-insufficient) tumor vessels maintain vessel stability, although tumor endothelium is commonly destabilized (17), which suggests that there are various types of tECs. However, the heterogeneity of tECs has not been studied extensively. The present study showed that strong Sox17 expression distinguished tEC populations with greater mitotic ability and higher surface VEGFR2 expression. Histological analysis also showed that high Sox17 expression correlated with VEGFR2 expression in tumor vessels. It is possible that Sox17high tECs might be more sensitive to VEGF and thus divide more rapidly than Sox $17^{\text {low }} t E C s$. In addition, there was a higher percentage of Sox17 high tECs in LLC tumors than in B16F10 tumors. These data suggest that the angiogenic properties of tumor vessels might be determined by the collective traits of the individual tECs. Interestingly, in the pool of postnatal HSCs, Sox17 expression distinguishes HSCs that have fetal properties (23). Our present results thus reiterate the importance of Sox 17 expression in subdividing cell populations. Taken together, our findings suggest a strong correlation between Sox 17 expression and the angiogenic potential within heterogeneous tECs. More studies are needed to characterize these heterogeneous tECs.

Excessive angiogenesis and vascular abnormality are typical features of many tumors $(4,32)$. While traditional antiangiogenic strategies have attempted to reduce tumor angiogenesis, both "vessel blocking" and "vessel normalization" are currently considered novel paradigms to complement existing antiangiogenic strategies (1). Hence, it is interesting that loss of PHD2 or of regulator of $\mathrm{G}$ protein signaling 5 (RGS5) results in normalization of tumor vessels, although their inhibition does not reduce vessel density $(17,33)$. Of particular note is that Sox 17 deletion simultaneously inhibited tumor angiogenesis and induced vascular normalization. More importantly, Sox 17 deletion induced prolonged normalization of abnormal tumor vessels, resulting in vessels with a phenotype resembling that of PHD2-insufficient tumor vessels and contrasting with the transient normalization elicited by current antiangiogenic therapy $(1,34)$. Because the upstream regulators of Sox17 expression in tECs are unknown, the question remains as to whether antiangiogenic therapy promotes vessel normalization by regulation of Sox 17 expression. As tumors progress to more severe grades, tumor vessels become increasingly abnormal (1). The changes in tumor vessel shape over time are well characterized, but little is known about whether a particular protein continuously regulates this remodeling. Our findings from the time-specific gene ablation experiments indicated that Sox17 exerts its effects throughout tumor angiogenesis. Our findings regarding the persistent expression and function of Sox 17 in tECs identified Sox17 as a persistent regulator that promotes tumor angiogenesis and destabilizes tumor vessels.

Inhibition of proangiogenic regulators reduces tumor growth. In this study, Sox17 deletion at the beginning of tumor growth consistently diminished tumor progression. There are several important points to consider regarding inhibition of tumor growth by Sox 17 deletion. First, the inhibition was transient only during early tumor growth - there was no growth inhibition when Sox17 was deleted during late tumor growth in the mouse model we used. Second, Sox17 deletion created a tumor environment that was favorable for tumor cell survival, as indicated by markedly reduced tumor necrosis. In contrast, when VEGF or Ang-2 are blocked, inhibited tumors show increased intratumoral necrosis mediated by hypoxia $(4,35,36)$. The tumoral features that develop in response to Sox 17 deletion might be attributed to reduced angiogenesis and improved vascular function. An oxygen-rich environment created by the loss of Sox 17 might delay tumor growth and metastasis by reducing the malignant and invasive characteristics of tumor cells (1); however, the same environment might fuel the proliferation of tumor cells by supplying oxygen and nutrients for anabolic processes. Despite its ambiguous effects on tumor cells, improved vascular function is advantageous when combined with chemotherapy, as it increases intratumoral delivery of anticancer agents (1). In fact, the anticancer drug cisplatin inhibited tumor growth more effectively after late Sox17 excision, illustrating that the improved vascular function subsequent to Sox17 deletion could be exploited for therapeutic purposes. Reduced tumor metastasis subsequent to late Sox 17 deletion might also be ascribed to decreased intravasation of tumor cells based on improved vascular integrity $(5,37)$. We cannot exclude the possibility that Sox17 expressed in tumor vessels within metastasized tumors might play a role in the growth of metastatic lesions. Taken together, our results suggest that Sox17 deletion in tumor vessels might help to inhibit tumor progression throughout tumor growth.

Although the importance of Sox17 in determining cell fate and maintaining cell identity has been reported in primitive cells, such as ventral foregut progenitors and HSCs $(22,23)$, its effect on specific cell behavior remains unknown. We found that Sox17 autonomously promoted sprouting of ECs by stimulating cell migration and proliferation at the expense of adherence to the ECM. These findings indicate that increased motility and cell division of ECs are the main cellular mechanisms underlying Sox17-mediated tumor angiogenesis. Thus, Sox 17 has a novel role in that it influences the cellular behaviors of differentiated effector cells.

Since Sox 17 is a transcription factor, its proangiogenic activity is most likely due to modulation of EC gene expression. Microarray analysis at the whole-transcriptome level revealed that Sox 17 depletion in primary cultured ECs changed the expression of a specific set of genes; global effects on transcription were not observed. Considering the effect of Sox 17 on tumor vessels, the downstream genes selectively regulated by Sox 17 might be key players in tumor angiogenesis. Indeed, Sox17 knockdown downregulated VEGFR2, which is well established as having an important function in tumor angiogenesis (38). However, regulation of VEGFR2 expression in tECs has not previously been characterized. We confirmed that VEGFR2 expression was reduced in Sox17-deficient tumor vessels and also observed that high Sox17 expression distinguished tECs with high VEGFR2 expression from the heterogeneous tEC population. Blockade of the VEGF pathway has an antiangiogenic effect (4), so reduced VEGFR2 expression in tECs as a result of Sox17 deletion might lead to reduced tumor angiogenesis and to normalization of tumor vessels. Unlike previous reports about vascular regression caused by VEGF blockade (39), Sox17 deletion 
did not cause vascular regression (data not shown). It is possible that Sox 17 deletion might incompletely block the VEGF pathway or affect the coordinated actions of multiple proteins, not just VEGFR2. Taken together, our findings indicate that Sox17 is a novel regulator of VEGFR2 expression in tumor angiogenesis.

Even though the VEGF pathway is the principal pathway in tumor angiogenesis, other mechanisms, such as the recruitment of inflammatory cells, have also been implicated in tumor angiogenesis $(4,5)$. In this regard, Sox 17 deletion reduced tumor-associated $\mathrm{CD} 11 \mathrm{~b}^{+} \mathrm{Gr}-1^{+}$myeloid cells, which induce tumor growth and tumor angiogenesis in a VEGF blockade-resistant manner $(28,29)$. Interestingly, CCL2, one of the genes most downregulated by Sox17 knockdown, has been implicated in immune cell recruitment into tumors (40). The detailed molecular mechanisms that underlie the increase in tumor-associated $\mathrm{CD} 11 \mathrm{~b}^{+} \mathrm{Gr}-1^{+}$myeloid cells after Sox17 deletion need to be investigated further. Our results suggest that Sox 17 might promote tumor angiogenesis, in part via a VEGF-independent mechanism, by recruiting inflammatory cells.

The microarray analysis identified many interesting genes as candidate genes that may act downstream of Sox17. CXCR7, the gene most downregulated by Sox 17 knockdown, is expressed in tECs and is important for tumor angiogenesis and tumor progression in several ways (41). ephrin-B2, which was also downregulated by Sox 17 knockdown, is well known for having a proangiogenic role in tumor angiogenesis $(42,43)$. We found that Sox 17 regulated the expression of many genes with wide-ranging functions. For example, genes for the deposition and modification of ECM and genes encoding secretory molecules and exocytosis proteins, such as DYNLL2, EXOC7, RhoQ, and Rab3B, might be involved in the circumferential effect of Sox17 on basement membrane and pericytes. Sox17-controlled cell-cell interactions and cell adhesion to ECM might be regulated by genes that encode membrane proteins, such as claudins, CD44, CD59, and syndecan 4. Genes involved in signaling pathways might coordinate endothelial behavior that is regulated by Sox17. Despite their importance in endothelial functions, the roles of these genes in Sox17-mediated tumor angiogenesis remain to be clarified. Notably, the list of genes regulated by Sox17 in ECs does not include Sox17-downstream genes identified in other cells. In colon carcinoma cells and oligodendrocyte progenitors $(44,45)$, Sox17 attenuates the expression of some Wnt target genes by antagonizing $\beta$-catenin. However, according to our microarray data, Sox17 did not seem to affect Wnt target genes in ECs. Gene expression induced by ectopic Sox17 in HSCs (46) did not overlap with the transcriptional changes induced by Sox17 in ECs. This inconsistency suggests that Sox 17 may regulate the transcription of different genes, depending on cell type.

In conclusion, our findings demonstrated that the transcription factor Sox17 is a persistent regulator of tumor angiogenesis. We provided evidence that heterogeneous tECs can be subdivided by Sox17 expression. Our results identified Sox 17 as a regulator of VEGFR2 expression in tECs and helped to elucidate the role of transcriptional regulation in tumor angiogenesis. There was strong and specific expression of Sox 17 in tECs, and Sox17 deletion inhibited tumor angiogenesis and normalized tumor vessels. Thus, Sox17 inhibition has potential value as part of an antiangiogenic approach to combine vessel blocking and vessel normalization.

\section{Methods}

Mouse lines. Sox17GFP/+ Sox17fl/+ (23), Cdh5(PAC)-CreER ${ }^{T 2}$ (43), and Tet-O-Sox17 (47) mice were housed in the Animal Facility at the Korea Advanced Insti- tute of Science and Technology. VE-cadherin-tTA mice (48) were provided by K. Alitalo (University of Helsinki, Helsinki, Finland). All animals were bred in a pathogen-free animal facility. $C d h 5(P A C)-C r e E R^{T 2}$ mice were crossed with Sox $17^{\ell / f l}$ mice to generate the mice used for the LOF studies. For Sox 17 deletion in ECs at the desired time points, mice were given intraperitoneal injections of $2 \mathrm{mg}$ tamoxifen (Sigma-Aldrich) on the indicated days. VE-cadherintTA mice were bred with Tet-O-Sox 17 mice to produce the mice used in the GOF studies. Mice were given $1 \mathrm{mg} / \mathrm{ml}$ tetracycline (Sigma-Aldrich) in their drinking water to prevent leaky expression of Sox 17. To induce Sox 17 overexpression in ECs, tetracycline was withdrawn on the indicated days.

Tumor models and treatment schedules. Mouse LLC and B16F10 melanoma cell lines that were syngeneic to the mouse models used in this study were obtained from the American Type Culture Collection. To generate tumor models, suspensions of LLC or B16F10 cells $\left(1 \times 10^{6}\right.$ cells in 100 $\mu \mathrm{l})$ were implanted subcutaneously in the flanks of mice. Tumor volume was measured every other day starting on day 6 after implantation. For the LLC tumor model, a chemotherapeutic agent $(10 \mathrm{mg} / \mathrm{kg}$ cisplatin; Sigma-Aldrich) was injected into the peritoneum once a week. Later, on the indicated days, mice were anesthetized by intramuscular injection of 80 $\mathrm{mg} / \mathrm{kg}$ ketamine and $12 \mathrm{mg} / \mathrm{kg}$ xylazine. Tumor mass was measured, and the primary tumor and lungs were harvested for further analysis.

Clinical specimens. Clinical specimens of brain tumors, including lowgrade glioma and high-grade glioblastoma, were obtained from the Department of Neurosurgery of Asan Medical Center.

Histological analysis. For H\&E staining, tumors and lungs were fixed overnight in $4 \%$ paraformaldehyde. After tissue processing using standard procedures, samples were embedded in paraffin and cut into 3- $\mu \mathrm{m}$ sections, followed by H\&E staining. For immunofluorescence studies, tumors were fixed in $1 \%$ paraformaldehyde in PBS, dehydrated in 30\% sucrose solution overnight, and embedded in tissue-freezing medium. Frozen blocks were cut into $60-\mu \mathrm{m}$ sections. To visualize tumor vessels, sections were stained with the appropriate antibodies, and fluorescent images were obtained by confocal microscopy. To assess vascular leakage from tumor vessels, extravasated dextran and Evans blue were measured. See Supplemental Methods for details.

Flow cytometry analysis and sorting of $t E C$. To examine Sox 17 and VEGFR2 expression at the single-cell level, single-cell suspensions (obtained by digestion of LLC or B16F10 tumors) were stained with the appropriate antibodies and analyzed using flow cytometry. To analyze the transcripts in tECs, CD $31^{+}$cells in tumors were enriched using microbeads, and CD $31^{+} \mathrm{CD} 45^{-}$Ter119- $\mathrm{tECs}$ were purified further by FACS. See Supplemental Methods for details.

Primary culture of mouse ECs. Initially, single cells were isolated from 3- to 6-day-old neonatal mouse lungs. After incubation for 2 days, ECs were enriched to at least $95 \%$ purity by MACS sorting using rat monoclonal anti-mouse CD102 antibody (Biolegend). Purified ECs were cultured further in EGM-2 medium, and the mitotic and migratory ability of primary ECs were measured. Sox 17 expression in primary ECs was confirmed by immunostaining (Supplemental Figure 2E) with an anti-Sox17 antibody (R\&D Systems). See Supplemental Methods for details.

Transcript analysis by quantitative PCR and microarray. Total RNA was isolated from tECs and tumor hematopoietic cells purified from tumors and from HUVECs that were treated with siRNAs against Sox17 (for Sox17 knockdown) or scrambled siRNAs (as a control). The total RNA was then used for quantitative real-time PCR or microarray analysis (Agilent human GE $4 \times 44 \mathrm{~K}$ [V2]). To establish the integrity of the RNA for the microarray analysis, we used Sox17-silenced HUVECs rather than purified tECs to obtain enough RNA, since there were very limited amounts of tECs. Microarray data are available at GEO (accession no. GSE37395; http:// www.ncbi.nlm.nih.gov/geo/query/acc.cgi?acc=GSE37395). See Supplemental Methods for details of transcript analysis. 
Statistics. Values are reported as mean \pm SD. Significant differences between means were determined by analysis of variance followed by 2 -tailed StudentNewman-Keuls test. A $P$ value less than 0.05 was considered significant.

Study approval. All patient samples were collected with written informed consent under protocols (KH2012-01 and 2011-1012) approved by the IRBs of the Korea Advanced Institute of Science and Technology and Asan Medical Center. Animal care and all animal procedures were approved by the Animal Care Committee of Korea Advanced Institute of Science and Technology.

\section{Acknowledgments}

This study was supported by grants from the Korean Health Technology R\&D Project (A084424 and A084697, to I. Kim) and the National R\&D Program for Cancer Control (1120030, to I. Kim) of the Ministry of Health and Welfare, Republic of Korea. We are grateful to S. Seo, E.S. Lee, and T.C. Yang for expert technical assistance.

Received for publication April 30, 2012, and accepted in revised form October 12, 2012.

Address correspondence to: Injune Kim, Graduate School of Medical Science and Engineering, Korea Advanced Institute of Science and Technology, Daejeon 305-701, Republic of Korea. Phone: 82.42.350.4238; Fax: 82.42.350.4240; E-mail: injunek@kaist.ac.kr.
1. Carmeliet P, Jain RK. Principles and mechanisms of vessel normalization for cancer and other angiogenic diseases. Nat Rev Drug Discov. 2011;10(6):417-427.

2. Hanahan D, Weinberg RA. Hallmarks of cancer: the next generation. Cell. 2011;144(5):646-674.

3. Koh YJ, et al. Double antiangiogenic protein, DAAP, targeting VEGF-A and angiopoietins in tumor angiogenesis, metastasis, and vascular leakage. Cancer Cell. 2010;18(2):171-184.

4. Chung AS, Ferrara N. Developmental and pathological angiogenesis. Annu Rev Cell Dev Biol. 2011; 27:563-584.

5. Saharinen P, Eklund L, Pulkki K, Bono P, Alitalo $\mathrm{K}$. VEGF and angiopoietin signaling in tumor angiogenesis and metastasis. Trends Mol Med. 2011; 17(7):347-362.

6. Bergers G, Hanahan D. Modes of resistance to anti-angiogenic therapy. Nat Rev Cancer. 2008; 8(8):592-603.

7. Ebos JM, Lee CR, Cruz-Munoz W, Bjarnason GA, Christensen JG, Kerbel RS. Accelerated metastasis after short-term treatment with a potent inhibitor of tumor angiogenesis. Cancer Cell. 2009;15(3):232-239.

8. Paez-Ribes M, et al. Antiangiogenic therapy elicits malignant progression of tumors to increased local invasion and distant metastasis. Cancer Cell. 2009; 15(3):220-231.

9. Casanovas $O$. The adaptive stroma joining the antiangiogenic resistance front. J Clin Invest. 2011; 121(4):1244-1247.

10. Kappel A, Ronicke V, Damert A, Flamme I, Risau W, Breier G. Identification of vascular endothelial growth factor (VEGF) receptor-2 (Flk-1) promoter/ enhancer sequences sufficient for angioblast and endothelial cell-specific transcription in transgenic mice. Blood. 1999;93(12):4284-4292.

11. Suchting S, et al. The Notch ligand Delta-like 4 negatively regulates endothelial tip cell formation and vessel branching. Proc Natl Acad Sci US A 2007;104(9):3225-3230.

12. Murakami M, et al. FGF-dependent regulation of VEGF receptor 2 expression in mice. J Clin Invest. 2011;121(7):2668-2678.

13. Wigle JT, Oliver G. Prox1 function is required for the development of the murine lymphatic system. Cell. 1999;98(6):769-778.

14. You LR, Lin FJ, Lee CT, DeMayo FJ, Tsai MJ, Tsai SY. Suppression of Notch signalling by the COUPTFII transcription factor regulates vein identity. Nature. 2005;435(7038):98-104.

15. Lee D, et al. ER71 acts downstream of BMP, Notch, and Wnt signaling in blood and vessel progenitor specification. Cell Stem Cell. 2008;2(5):497-507.

16. Gerhardt H, et al. VEGF guides angiogenic sprouting utilizing endothelial tip cell filopodia. J Cell Biol. 2003;161(6):1163-1177.
17. Mazzone M, et al. Heterozygous deficiency of PHD2 restores tumor oxygenation and inhibits metastasis via endothelial normalization. Cell. 2009; 136(5):839-851.

18. Eilken HM, Adams RH. Dynamics of endothelial cell behavior in sprouting angiogenesis. Curr Opin Cell Biol. 2010;22(5):617-625.

19. Felcht M, et al. Angiopoietin-2 differentially regulates angiogenesis through TIE2 and integrin signaling. J Clin Invest. 2012;122(6):1991-2005.

20. Schepers GE, Teasdale RD, Koopman P. Twenty pairs of sox: extent, homology, and nomenclature of the mouse and human sox transcription factor gene families. Dev Cell. 2002;3(2):167-170.

21. Kanai-Azuma M, et al. Depletion of definitive gut endoderm in Sox17-null mutant mice. Development. 2002;129(10):2367-2379.

22. Spence JR, et al. Sox17 regulates organ lineage segregation of ventral foregut progenitor cells. Dev Cell. 2009;17(1):62-74.

23. Kim I, Saunders TL, Morrison SJ. Sox17 dependence distinguishes the transcriptional regulation of fetal from adult hematopoietic stem cells. Cell. 2007;130(3):470-483.

24. Burtscher I, Barkey W, Schwarzfischer M, Theis FJ, Lickert H. The Sox17-mCherry fusion mouse line allows visualization of endoderm and vascular endothelial development. Genesis. 2011;50(6):496-505.

25. Matsui T, et al. Redundant roles of Sox 17 and Sox 18 in postnatal angiogenesis in mice. J Cell Sci. 2006; 119(pt 17):3513-3526.

26. Bilguvar K, et al. Susceptibility loci for intracranial aneurysm in European and Japanese populations. Nat Genet. 2008;40(12):1472-1477.

27. Charo IF, Ransohoff RM. The many roles of chemokines and chemokine receptors in inflammation. N Engl J Med. 2006;354(6):610-621.

28. Shojaei $F$, et al. Tumor refractoriness to anti-VEGF treatment is mediated by CD $11 \mathrm{~b}+\mathrm{Gr} 1+$ myeloid cells. Nat Biotechnol. 2007;25(8):911-920.

29. Shojaei F, et al. G-CSF-initiated myeloid cell mobilization and angiogenesis mediate tumor refractoriness to anti-VEGF therapy in mouse models. Proc Natl Acad Sci U S A. 2009;106(16):6742-6747.

30. Jain RK, di Tomaso E, Duda DG, Loeffler JS, Sorensen AG, Batchelor TT. Angiogenesis in brain tumours. Nat Rev Neurosci. 2007;8(8):610-622.

31. Adams RH, Alitalo K. Molecular regulation of angiogenesis and lymphangiogenesis. Nat Rev Mol Cell Biol. 2007;8(6):464-478.

32. Pasqualini R, Arap W, McDonald DM. Probing the structural and molecular diversity of tumor vasculature. Trends Mol Med. 2002;8(12):563-571.

33. Hamzah J, et al. Vascular normalization in Rgs5deficient tumours promotes immune destruction. Nature. 2008;453(7193):410-414.
34. Goel S, et al. Normalization of the vasculature for treatment of cancer and other diseases. Physiol Rev. 2011;91(3):1071-1121.

35. Augustin HG, Koh GY, Thurston G, Alitalo K. Control of vascular morphogenesis and homeostasis through the angiopoietin-Tie system. Nat Rev Mol Cell Biol. 2009;10(3):165-177.

36. Mazzieri R, et al. Targeting the ANG2/TIE2 axis inhibits tumor growth and metastasis by impairing angiogenesis and disabling rebounds of proangiogenic myeloid cells. Cancer Cell. 2011;19(4):512-526.

37. Cooke VG, et al. Pericyte depletion results in hypoxia-associated epithelial-to-mesenchymal transition and metastasis mediated by met signaling pathway. Cancer Cell. 2012;21(1):66-81.

38. Millauer B, Shawver LK, Plate KH, Risau W, Ullrich A. Glioblastoma growth inhibited in vivo by a dominant-negative Flk-1 mutant. Nature. 1994; 367(6463):576-579.

39. Inai $\mathrm{T}$, et al. Inhibition of vascular endothelial growth factor (VEGF) signaling in cancer causes loss of endothelial fenestrations, regression of tumor vessels, and appearance of basement membrane ghosts. Am J Pathol. 2004;165(1):35-52.

40. Izhak L, Wildbaum G, Jung S, Stein A, Shaked Y, Karin N. Dissecting the autocrine and paracrine roles of the CCR2-CCL2 axis in tumor survival and angiogenesis. PLoS One. 2012;7(1):e28305.

41. Miao Z, et al. CXCR7 (RDC1) promotes breast and lung tumor growth in vivo and is expressed on tumor-associated vasculature. Proc Natl Acad Sci US A. 2007;104(40):15735-15740.

42. Sawamiphak S, et al. Ephrin-B2 regulates VEGFR2 function in developmental and tumour angiogenesis. Nature. 2010;465(7297):487-491.

43. Wang Y, et al. Ephrin-B2 controls VEGF-induced angiogenesis and lymphangiogenesis. Nature. 2010; 465(7297):483-486.

44. Sinner D, et al. Sox 17 and Sox 4 differentially regulate beta-catenin/T-cell factor activity and proliferation of colon carcinoma cells. Mol Cell Biol. 2007;27(22):7802-7815.

45. Chew LJ, et al. SRY-box containing gene 17 regulates the Wnt/beta-catenin signaling pathway in oligodendrocyte progenitor cells. J Neurosci. 2011; 31(39):13921-13935.

46. He S, Kim I, Lim MS, Morrison SJ. Sox17 expression confers self-renewal potential and fetal stem cell characteristics upon adult hematopoietic progenitors. Genes Dev. 2011;25(15):1613-1627.

47. Park KS, Wells JM, Zorn AM, Wert SE, Whitsett JA. Sox 17 influences the differentiation of respiratory epithelial cells. Dev Biol. 2006;294(1):192-202.

48. Sun JF, et al. Microvascular patterning is controlled by fine-tuning the Akt signal. Proc Natl Acad SciU S A. 2005;102(1):128-133. 